\title{
Correction of Radar Reflectivity and Differential Reflectivity for Rain Attenuation at $X$ Band. Part II: Evaluation and Application
}

\author{
S.-G. Park, M. Maki, And K. Iwanami \\ National Research Institute for Earth Science and Disaster Prevention, Tsukuba, Japan \\ V. N. BRINGI AND V. CHANDRASEKAR \\ Department of Electrical and Computer Engineering, Colorado State University, Fort Collins, Colorado
}

(Manuscript received 25 February 2005, in final form 7 April 2005)

\begin{abstract}
In this paper, the attenuation-correction methodology presented in Part I is applied to radar measurements observed by the multiparameter radar at the X-band wavelength (MP-X) of the National Research Institute for Earth Science and Disaster Prevention (NIED), and is evaluated by comparison with scattering simulations using ground-based disdrometer data. Further, effects of attenuation on the estimation of rainfall amounts and drop size distribution parameters are also investigated. The joint variability of the corrected reflectivity and differential reflectivity show good agreement with scattering simulations. In addition, specific attenuation and differential attenuation, which are derived in the correction procedure, show good agreement with scattering simulations. In addition, a composite rainfall-rate algorithm is proposed and evaluated by comparison with eight gauges. The radar-rainfall estimates from the uncorrected (or observed) $Z_{\mathrm{H}}$ produce severe underestimation, even at short ranges from the radar and for stratiform rain events. On the contrary, the reflectivity-based rainfall estimates from the attenuation-corrected $Z_{\mathrm{H}}$ does not show such severe underestimation and does show better agreement with rain gauge measurements. More accurate rainfall amounts can be obtained from a simple composite algorithm based on specific differential phase $K_{\mathrm{DP}}$, with the $R\left(Z_{\mathrm{H}_{-}}\right.$cor $)$estimates being used for low rainfall rates $\left(K_{\mathrm{DP}} \leq 0.3^{\circ} \mathrm{km}^{-1}\right.$ or $Z_{\mathrm{H}_{-}}$cor $\leq$ $35 \mathrm{dBZ}$ ). This improvement in accuracy of rainfall estimation based on $K_{\mathrm{DP}}$ is a result of the insensitivity of the rainfall algorithm to natural variations of drop size distributions (DSDs). The $Z_{\mathrm{H}}, Z_{\mathrm{DR}}$, and $K_{\mathrm{DP}}$ data are also used to infer the parameters (median volume diameter $D_{0}$ and normalized intercept parameter $N_{w}$ ) of a normalized gamma DSD. The retrieval of $D_{0}$ and $N_{w}$ from the corrected radar data show good agreement with those from disdrometer data in terms of the respective relative frequency histograms. The results of this study demonstrate that high-quality hydrometeorological information on rain events such as rainfall amounts and DSDs can be derived from X-band polarimetric radars.
\end{abstract}

\section{Introduction}

Park et al. (2005, hereafter Part I) dealt with characteristic properties of empirical relations among polarimetric variables at the X-band wavelength for attenuation correction using the self-consistent method with constraints. In this paper, the attenuation-correction methodology is modified for the X-band wavelength, based on the results obtained from scattering simula-

Corresponding author address: Dr. Masayuki Maki, Advanced Technology Research Group, National Research Institute for Earth Science and Disaster Prevention, 3-1 Tennodai, Tsukuba, Ibaraki 305-0006, Japan.

E-mail: maki@bosai.go.jp tions in Part I, and is evaluated with real radar measurements observed by a multiparameter radar at $\mathrm{X}$ band (MP-X) of the National Research Institute for Earth Science and Disaster Prevention (NIED) of Japan.

Most previous studies accounting for correction resulting from rain attenuation were focused on rainfall estimation, rather than validation of the correction methodology (e.g., Ryzhkov and Zrnic 1995; Matrosov et al. 2002; Iwanami et al. 2003). In this study, emphasis is placed on the evaluation of the correction method by comparing the corrected reflectivity $Z_{\mathrm{H}}$ and differential reflectivity $Z_{\mathrm{DR}}$ with the values obtained from scattering simulations using ground-based disdrometer data. Then, effects of attenuation on rainfall estimation and 
drop size distribution (DSD) retrieval are also investigated. One final goal of this study is to demonstrate that valuable hydrometeorological information, such as rainfall amounts and DSDs, can be extracted from the $\mathrm{X}$-band polarimetric measurements if correction for attenuation is done accurately.

The data used for validating the attenuationcorrection methodology are presented in section 2. In section 3, the modification of the self-consistent method to the MP-X radar is described, based on the results from scattering simulations in Part I. The modified correction method is evaluated in section 4, by comparing with scattering simulations using groundbased disdrometer data. Consistencies among the corrected radar data are also analyzed by comparison with the empirical relations among the polarimetric variables obtained from scattering simulations. Section 5 presents the effects of attenuation on the estimation of rainfall amounts and DSDs. The rainfall amounts and DSDs are estimated from the MP-X radar data before and after correction for rain attenuation and the results are compared with rain gauge and disdrometer measurements. The results are summarized in section 6 .

\section{Data sources}

\section{a. Radar measurements}

The MP-X radar of NIED was completed in the year 2000 for hydrological and meteorological applications (Iwanami et al. 2001). Figure 1 shows the exterior of the MP-X radar. The radar is mounted on a 4-ton truck and is easily movable. The basic characteristics of the MP-X radar are presented in Table 1 . The radar operates at a frequency of $9.375 \mathrm{GHz}$ and simultaneously transmits equal power in the horizontal and vertical channels (i.e., slant $45^{\circ}$ transmission) and simultaneously re-

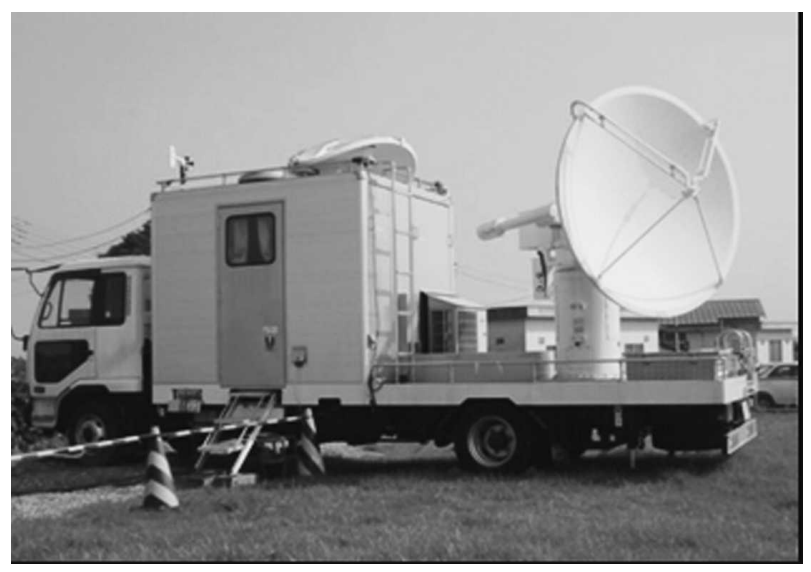

FIG. 1. Photograph of the MP-X radar.
TABLE 1. System characteristics of the MP-X radar.

\begin{tabular}{|c|c|}
\hline Frequency & $9.375 \mathrm{GHz}$ \\
\hline Antenna type & Circular parabola, $2.1 \mathrm{m \phi}$ \\
\hline \multicolumn{2}{|l|}{ Scan range (scan rate): } \\
\hline Azimuth & Full circle (same) \\
\hline Elevation & $-2^{\circ}$ to $+92^{\circ}$ (same) \\
\hline Antenna gain & $41.6 \mathrm{~dB}$ \\
\hline Beamwidth & $1.3^{\circ}$ \\
\hline $\begin{array}{l}\text { Receiver dynamic } \\
\text { range }\end{array}$ & $83 \mathrm{~dB}$ \\
\hline Transmitter tube & Magnetron \\
\hline Peak power & $50 \mathrm{~kW}$ \\
\hline Pulse length & $0.5 \mu \mathrm{s}$ \\
\hline $\begin{array}{l}\text { Pulse-repetition } \\
\text { frequency }\end{array}$ & $\leq 1800 \mathrm{~Hz}$ \\
\hline Polarization & Horizontal and vertical \\
\hline Doppler processing & PPP, FFT \\
\hline Noise figure & $2.3 \mathrm{~dB}$ \\
\hline $\begin{array}{l}\text { Minimum detectable } \\
\text { signal }\end{array}$ & $-110 \mathrm{dBm}$ \\
\hline Observation range & $80 \mathrm{~km}$ \\
\hline Variables available & $\begin{array}{l}\text { Reflectivity at horizontal polarization } \\
Z_{\mathrm{H}} \text {, differential reflectivity } Z_{\mathrm{DR}} \\
\text { total differential phase } \Psi_{\mathrm{DP}}, \\
\text { copolar correlation coefficient } \rho_{\mathrm{HV}}, \\
\text { Doppler velocity and spectral width }\end{array}$ \\
\hline
\end{tabular}

ceives the horizontally and vertically polarized scattered signals with two receivers. The available polarimetric variables include radar reflectivity at the horizontal polarization $Z_{\mathrm{H}}$, differential reflectivity $Z_{\mathrm{DR}}$, total differential phase $\Psi_{\mathrm{DP}}$, and copolar correlation coefficient $\rho_{\mathrm{HV}}$, as well as Doppler velocity and spectral width.

The MP-X radar was first operated from June to October 2001 , in the Tsukuba area $\left(36.1^{\circ} \mathrm{N}, 140.2^{\circ} \mathrm{E}\right)$ of Japan. These first observations were used to evaluate the quality of the polarimetric measurements and to develop an algorithm for rainfall estimation based on the $K_{\mathrm{DP}}$ data. For providing ground truth data, three disdrometers (Joss-Waldvogel type) were additionally set up at three different ranges along an azimuth angle of $294^{\circ}$. The number of drops counted by each disdrometer was averaged every minute. Since 2003, the MP-X radar has been operated at Ebina $\left(35.4^{\circ} \mathrm{N}, 139.4^{\circ} \mathrm{E}\right)$, Japan, in order to test its operational use for monitoring and forecasting heavy rainfall over mountainous and flat metropolitan areas, which frequently experience flood and landslide disasters caused by heavy rainfall (Maki et al. 2005). For the validation of radar-rainfall estimates, two different rain gauge networks were set up (Fig. 2). The first network consisted of four rain gauges of a tipping-bucket type placed along an azimuth angle of about $257^{\circ}$, at approximate $10-\mathrm{km}$ intervals from the MP-X radar. The second network, which also consisted of four rain gauges, was arranged within 


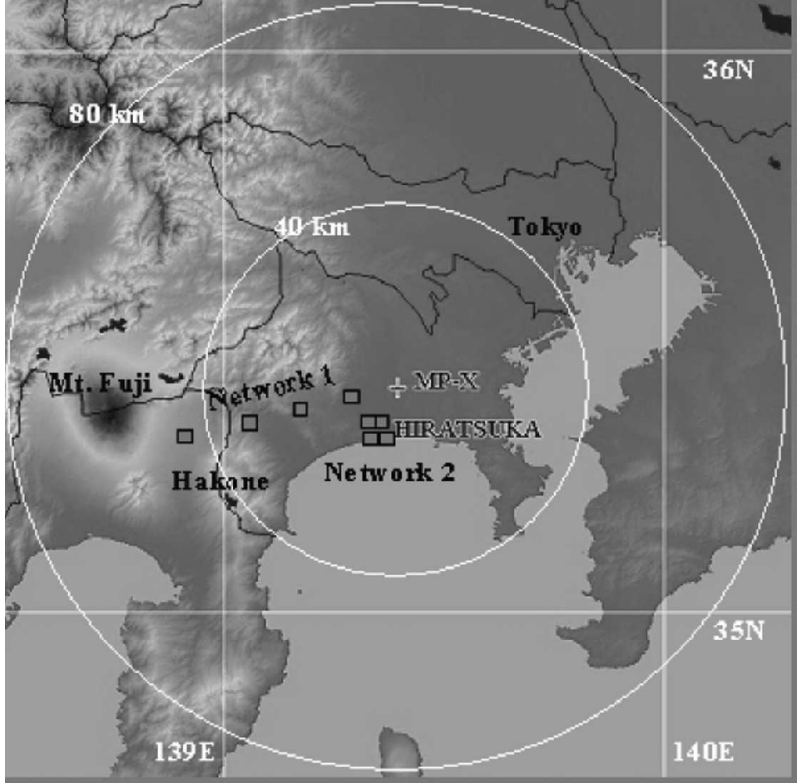

FIG. 2. Map showing the relative locations of the MP-X radar $(+)$ and the rain gauge networks $(\square)$ during the observation in 2003.

an area of about $1 \mathrm{~km}^{2}$ at Hiratsuka, Japan, located at an azimuth angle of about $205^{\circ}$ and at a range of about $10 \mathrm{~km}$ from the MP-X radar. These eight rain gauges recorded 1-min rainfall amounts with a resolution of $0.1 \mathrm{~mm}$.

Since 2001 the MP-X radar has observed various precipitation systems, such as typhoons, midlatitude thunderstorms, and stationary front systems. In this study, a typhoon case observed on 11 September 2001 was analyzed for evaluating the method for attenuation correction. Then, the correction method was applied to three rain events during observations in 2003 for investigating the effect of attenuation on rainfall estimation. The three rain events in 2003 consisted of two stratiform events (1700-2400 LST 25 July and 0000-1500 LST 15 August) associated with stationary front systems, and one convective event (0400-1300 LST 9 August) during the passage of a typhoon.

During passage of the typhoon in 2001, the MP-X radar was operated in plan-position indicator (PPI) mode at a fixed elevation angle of $2.5^{\circ}$, which avoids ground clutter contamination. The number of samples for measuring the polarimetric variables was 256 , with a range resolution of $100 \mathrm{~m}$ and an azimuthal resolution sampling of $0.5^{\circ}$. The pulse-repetition frequency was $1800 \mathrm{~Hz}$ and the pulse-pair processing (PPP) algorithm was employed for the Doppler signal processing. During the three rain events in 2003, the observation mode of the MP-X radar was altered slightly. The polarimetric variables were collected by consecutive volume scans consisting of 11 elevation angles from $0.9^{\circ}$ to $21.5^{\circ}$, with the number of samples set at 100 and an azimuthal resolution of $1^{\circ}$. Among the 11 elevation angles, the data on the PPI surface of the third elevation angle $\left(2.1^{\circ}\right)$ were used for rainfall estimation, because that elevation angle is the lowest angle that is not affected by beam blockage or ground clutter over all eight rain gauges. PPI scans at the elevation angle of $2.1^{\circ}$ were repeated every $3 \mathrm{~min}$ by the scan scheduler.

\section{b. Preprocessing of the polarimetric radar measurements}

Before retrieving the hydrometeorological parameters from MP-X radar data, the polarimetric variables measured by the radar were processed by filtering algorithms, in order to eliminate "high frequency" random fluctuations from gate to gate. First, $Z_{\mathrm{H}}, Z_{\mathrm{DR}}$, and $\rho_{\mathrm{HV}}$ were processed by the infinite-impulse response (IIR) filter of Hubbert et al. (1993). The total differential phase $\Psi_{\text {DP }}$ range profiles were iteratively filtered in the range following the finite-impulse response (FIR) filter of Hubbert and Bringi (1995), in order to separate the scattering differential phase $(\delta)$ from the $\Psi_{\text {DP }}$ profiles and then extract the filtered differential propagation phase $\left(\phi_{\mathrm{DP}}\right)$. Once the filtered $\phi_{\mathrm{DP}}$ range profiles were extracted, the specific differential phase $K_{\mathrm{DP}}$ was then computed by a least squares fit to a varying number of consecutive range samples with respect to the attenuation-corrected reflectivity factor (10 samples if $Z_{\mathrm{H}}>45 \mathrm{dBZ}, 20$ samples for $35<Z_{\mathrm{H}}<5 \mathrm{dBZ}$, and 30 samples for $\left.Z_{\mathrm{H}}<35 \mathrm{~dB} Z\right)$. Such "telescoping" further smoothes $K_{\mathrm{DP}}$, especially for lighter rain segments.

To determine statistical errors (accuracies) of the polarimetric variables, the MP-X radar was sometimes operated at vertical incidence with radar antenna rotated by an integral multiple of full $360^{\circ}$ when widespread rain occurred over the radar site. This technique is based on Liu et al. (1993). The data from the vertical incidence observations were averaged over height intervals (below the melting level) characterized by high and stable $\rho_{\mathrm{HV}}$ values $(>0.98)$ and by stable $Z_{\mathrm{H}}$ values around $35 \mathrm{dBZ}$. The averaged values of each polarimetric variable was very stable with standard deviations of about $1 \mathrm{dBZ}, 0.25 \mathrm{~dB}, 0.01$, and $4^{\circ}$ for $Z_{\mathrm{H}}, Z_{\mathrm{DR}}, \rho_{\mathrm{HV}}$, and $\phi_{\mathrm{DP}}$, respectively. Using these vertical incidence observations, the accuracy of $K_{\mathrm{DP}}$ was estimated to be about $0.3^{\circ} \mathrm{km}^{-1}$ (note that the mean $K_{\mathrm{DP}}$ is $0^{\circ} \mathrm{km}^{-1}$ at vertical incidence). It is worthwhile to note that the accuracy of $K_{\mathrm{DP}}$ (i.e., $0.3^{\circ} \mathrm{km}^{-1}$ ) is the statistical error based on analysis of experimental data, not on an analytical model of errors. This experimentally based error of $K_{\mathrm{DP}}$ is different from errors based on an analytical model such as described by Gorgucci et al. (1999) or Ryzhkov and Zrnic (1996). Using the measurement er- 
TABLE 2. The NE and NB of rainfall accumulations for three different time intervals (15 min, $1 \mathrm{~h}$, and $3 \mathrm{~h}$ ) obtained from the radar-rainfall estimates $R\left(Z_{\mathrm{H} \_}\right.$uncor $), R\left(Z_{\mathrm{H}-}\right.$ cor $)$, and $R\left(K_{\mathrm{DP}}\right)$.

\begin{tabular}{|c|c|c|c|c|c|}
\hline & & \multirow[b]{3}{*}{$R\left(Z_{\mathrm{H}-\text { uncor }}\right)$} & \multirow[b]{3}{*}{$R\left(Z_{\mathrm{H} \_}\right.$cor $)$} & \multirow{2}{*}{\multicolumn{2}{|c|}{$\begin{array}{c}R\left(K_{\mathrm{DP}}\right) \\
\text { When } K_{\mathrm{DP}} \leq 0.3^{\circ} \mathrm{km}^{-1} \text { or } \\
Z_{\mathrm{H} \_} \text {cor } \leq 35 \mathrm{dBZ}\end{array}$}} \\
\hline & & & & & \\
\hline & & & & $R\left(Z_{\mathrm{H} \_}\right.$uncor $)$ & $R\left(Z_{\mathrm{H}-}\right.$ cor $)$ \\
\hline \multirow[t]{2}{*}{$15 \mathrm{~min}$} & NE (\%) & 50.9 & 25.9 & 23.9 & 21.1 \\
\hline & NB (\%) & -48.3 & -3.5 & -13.9 & -2.9 \\
\hline \multirow[t]{2}{*}{$1 \mathrm{~h}$} & NE (\%) & 48.9 & 19.1 & 18.4 & 14.8 \\
\hline & NB (\%) & -47.6 & -2.0 & -12.2 & -1.1 \\
\hline \multirow[t]{2}{*}{$3 \mathrm{~h}$} & NE (\%) & 47.9 & 15.8 & 15.6 & 11.4 \\
\hline & NB (\%) & -47.5 & -1.9 & -12.2 & -1.0 \\
\hline
\end{tabular}

ror of $\phi_{\mathrm{DP}}\left(4^{\circ}\right)$, the analytically based standard deviation of $K_{\mathrm{DP}}$ reaches about $2.2,0.7$, and $0.4^{\circ} \mathrm{km}^{-1}$ with the pathlengths used in the $K_{\mathrm{DP}}$ calculations of 1,2 , and $3 \mathrm{~km}$, respectively. For the vertical incidence observations used in this study, however, most data (>90\%) had standard deviations smaller than $0.3^{\circ} \mathrm{km}^{-1}$, under the same methodology as described above (namely, the same filtering and least squares fit schemes).

The value of $0.3^{\circ} \mathrm{km}^{-1}$ is appropriate for the estimation error of $K_{\mathrm{DP}}$ for the data used in this study, in particular, for rainfall estimation. The experiments of rainfall estimation based on $K_{\mathrm{DP}}$, for example, showed that an increase of threshold of $K_{\mathrm{DP}}$ that are applicable to rainfall estimation (e.g., 0.6 and $1.0^{\circ} \mathrm{km}^{-1}$ corresponding to two and about three times $0.3^{\circ} \mathrm{km}^{-1}$, respectively) did not the improve the accuracy of the rainfall estimates for rain events in 2003. Rather, an increase of the threshold of $K_{\mathrm{DP}}$ increased the error of the rainfall estimates, larger than those using the threshold of $0.3^{\circ} \mathrm{km}^{-1}$, which will be presented in Table 2. Note that Matrosov et al. (2002) used a threshold value of $0.1^{\circ} \mathrm{km}^{-1}$ for applying the $R-K_{\mathrm{DP}}$ relation.

The vertical incidence observations were also used for determining the differential system gain bias for correcting the $Z_{\mathrm{DR}}$ system bias (Gorgucci et al. 1999). In 2001 , the $Z_{\mathrm{DR}}$ system bias was estimated to be -1.38 dB (i.e., measured $Z_{\mathrm{DR}}$ values were to be increased by $1.38 \mathrm{~dB})$. In 2003, the $Z_{\mathrm{DR}}$ bias was estimated to be $-1.2 \mathrm{~dB}$. For determining the system $Z_{\mathrm{H}}$ bias (i.e., adjustment to the nominally established radar constant used to convert measured signal power to the effective reflectivity factor), we compared the measured $Z_{\mathrm{H}}$ by the radar with the simulated values using disdrometer data in very light rainfall where attenuation was negligible. The bias derived from these comparisons was then confirmed by the self-consistent technique using empirical $K_{\mathrm{DP}}-Z_{\mathrm{H}}$ and $K_{\mathrm{DP}}-\left(Z_{\mathrm{H}}, Z_{\mathrm{DR}}\right)$ relations (Gorgucci et al. 1999). The derived $Z_{\mathrm{H}}$ biases during the 2001 and 2003 observations were +5.5 and $-1.5 \mathrm{~dB}$, respectively. These adjustments to the "raw" $Z_{\mathrm{H}}$ and $Z_{\mathrm{DR}}$ data were performed prior to range filtering and attenuation correction.

As mentioned earlier, the MP-X radar is operated under the hybrid mode (Bringi and Chandrasekar 2001). Under this transmission mode, the polarimetric measurements are not at a pure orthogonal linear basis. Namely, the received signals at horizontal and vertical channels are not copolar to those transmitted at slant $45^{\circ}$. Compared to the alternating transmission of horizontally and vertically polarized waves that have been used generally as an orthogonal linear polarization, the slant $45^{\circ}$ transmission has several advantages, such as direct correlation measurement at zero lag, noncontamination by Doppler effects, higher accuracy of the polarimetric measurements for a given dwell time, and the capability of a higher scan speed (Holt et al. 1999; Doviak et al. 2000). However, if the mean canting angle is not zero, the polarimetric measurements at the slant $45^{\circ}$ transmission can be biased from those at the orthogonal linear transmission. As shown by Holt et al. (1999) and Matrosov et al. (2002), the differences, however, are small enough to be negligible. Holt et al. (1999) showed from experiment data that the polarimetric measurements $Z_{\mathrm{DR}}, \phi_{\mathrm{DP}}$, and $\rho_{\mathrm{HV}}$ at the slant $45^{\circ}$ transmission agreed well with those at the alternating transmission. Matrosov et al. (2002) showed from scattering simulations that the difference between $Z_{\mathrm{DR}}$ at the slant $45^{\circ}$ transmission and its true value at the orthogonal linear transmission is less than the generally expected accuracy of $Z_{\mathrm{DR}}$ measurements (about $0.2-$ $0.25 \mathrm{~dB}$ ). For most practical rain cases where the mean canting angle is expected to be zero, the increase (decrease) of canting angle from its mean value causes underestimation (overestimation) of $Z_{\mathrm{DR}}$ at the slant $45^{\circ}$ transmission. Based on these results, in this paper the polarimetric measurements are assumed to be equal to those obtained using the orthogonal linear polarization scheme. 


\section{Modification of the self-consistent method to MP-X radar data}

Based on the results obtained from the scattering simulations at the X-band wavelength in Part I of this study, the self-consistent method proposed and evaluated at $\mathrm{C}$ band by Bringi et al. (2001) was modified for the MP-X radar of NIED as follows: first, the $A_{\mathrm{H}}-K_{\mathrm{DP}}$ and $A_{\mathrm{DP}}-A_{\mathrm{H}}$ relations were assumed to be described by linear forms, though their exponents $c$ and $d$ increase above unity with temperature. For selecting an optimal value for the coefficient $\alpha$ of the $A_{\mathrm{H}}-K_{\mathrm{DP}}$ relation, the range from 0.025 to $0.575 \mathrm{~dB}\left({ }^{\circ}\right)^{-1}$ with an interval of 0.025 was used, which is an extension including the range (0.139-0.335) that is obtained from the scattering simulations in Part I. This range will be evaluated later with MP-X radar data by analyzing frequency of occurrences of optimal $\alpha$ values. For the exponent $b$ of the $A_{\mathrm{H}}-Z_{\mathrm{H}}$ relation, its mean value 0.780 at the temperature of $15^{\circ} \mathrm{C}$ was used in the $Z_{\mathrm{H}}$ correction procedure, based on the result in Part I that the exponent $b$ does not depend on drop shapes and has an almost constant value for a given temperature. In the $Z_{\mathrm{DR}}$ correction, the following relation was used for the constraint $Z_{\mathrm{DR}}\left(r_{0}\right)$ in the procedure for selecting optimal $\gamma$ :

$$
\begin{aligned}
& Z_{\mathrm{DR}}\left(r_{0}\right) \\
& =\left\{\begin{array}{lll}
0 & \text { when } & Z_{\mathrm{H}}\left(r_{0}\right) \leq 10 \mathrm{dBZ} \\
0.051 Z_{\mathrm{H}}-0.486 & \text { when } & 10<Z_{\mathrm{H}}\left(r_{0}\right) \leq 55 \mathrm{~dB} Z \\
2.3 & \text { when } & Z_{\mathrm{H}}\left(r_{0}\right)>55 \mathrm{dBZ}
\end{array}\right.
\end{aligned}
$$

(where $Z_{\mathrm{DR}}$ is in $\mathrm{dB}$ and $Z_{\mathrm{H}}$ is in $\mathrm{dB} Z$ ). Note that $Z_{\mathrm{H}}$ in the above relation must first be corrected for attenuation effects. The above relation is a mean relation obtained from the scattering simulations for the three different drop shapes described in Part I, at the temperature of $15^{\circ} \mathrm{C}$ and elevation angle of $0^{\circ}$. Note that it was shown in Part I that the $Z_{\mathrm{DR}}-Z_{\mathrm{H}}$ relation was nearly constant with respect to variation of temperature. In the present study, the radar data were obtained at low elevation angles of $2.1^{\circ}$ and $2.5^{\circ}$. If data are obtained at higher elevation angles, an elevation angle adjustment can be done, assuming Rayleigh scattering, and this adjustment can also be done for $K_{\mathrm{DP}}$ in the $A_{\mathrm{H}}-K_{\mathrm{DP}}$ relation for higher elevation angles.

The modified self-consistent method was applied to rays where the total change of differential propagation phase $\left(\Delta \phi_{\mathrm{DP}}\right)$ over a rain cell was larger than $10^{\circ}$. Otherwise, when $\Delta \phi_{\mathrm{DP}} \leq 10^{\circ}$, a fixed a priori value for $\alpha$ was used, instead of selection of an optimal $\alpha$ value. For these cases, the $Z_{\mathrm{H}}$ correction was accomplished by a simple correction method that determines $A_{\mathrm{H}}$ from the relation $A_{\mathrm{H}}=0.275 K_{\mathrm{DP}}$, whose coefficient is the modal value that will be shown later in analysis of the occurrence frequency of optimal $\alpha$. The $Z_{\mathrm{DR}}$ correction for cases with $\Delta \phi_{\mathrm{DP}} \leq 10^{\circ}$ was performed simply by using the relation $A_{\mathrm{DP}}=0.029 K_{\mathrm{DP}}$, instead of the selection of optimal $\gamma$ of the $A_{\mathrm{DP}}-A_{\mathrm{H}}$ relation. The coefficient $\left[0.029 \mathrm{~dB}\left({ }^{\circ}\right)^{-1}\right]$ of the $A_{\mathrm{DP}}-K_{\mathrm{DP}}$ relation is a mean value obtained from scattering simulations under the various conditions.

Because the above procedure for correcting attenuation is only valid for rain attenuation, it is necessary to detect the rain cell segment in each ray (i.e., detection of $r_{1}$ and $r_{0}$ ). Rain cells generally have higher $\rho_{\mathrm{HV}}$ values $(\geq 0.98)$ with relatively smaller variations as compared with ground clutter (Ryzhkov and Zrnic 1998). However, the MP-X radar operating at X band sometimes loses the signal resulting from excessive attenuation behind a strong rain echo, with a significant decrease of $\rho_{\mathrm{HV}}$ and the signal-to-noise ratio. In this study, the starting range of a rain call $\left(r_{1}\right)$ was determined using the standard deviation of $\phi_{\mathrm{DP}}$ less than or equal to $10^{\circ}$ and $\rho_{\mathrm{HV}}>0.7$ over 10 consecutive ranges $(1-\mathrm{km}$ range sector). The threshold value of $\rho_{\mathrm{HV}}(0.7)$ for detecting rain cells is the value that is generally accepted for rejecting ground clutter (Ryzhkov and Zrnic 1998). When the radar beam intercepts the bright band, the rain cell was limited up to a range with $\rho_{\mathrm{HV}}>0.94$ and below a height of $4.2 \mathrm{~km}$, which are thresholds for detecting bright bands based on analyses of $\rho_{\mathrm{HV}}$ fields in the MP-X radar observations. The determination of the ending range of a rain cell $\left(r_{0}\right)$ was determined using the standard deviation of $\phi_{\mathrm{DP}}$ larger than $10^{\circ}$ and $\rho_{\mathrm{HV}}$ $<0.7$ over five consecutive ranges $(0.5-\mathrm{km}$ range sector), together with a height constraint of $4.2 \mathrm{~km}$.

\section{Validation of attenuation correction}

On 11 September 2001, Typhoon 0115, embedding strong rainbands, passed near the MP-X radar site. Associated with the passage of the typhoon, a strong rainband moved from southwest to northeast during 01000300 LST, and severe attenuation was observed near 0200 LST. This typhoon case was analyzed for evaluating the modified self-consistent method for correcting attenuation. The corrected $Z_{\mathrm{H}}$ and $Z_{\mathrm{DR}}$ were compared with the simulated values based on the disdrometer DSD data for validation.

\section{a. An example of severe attenuation}

Figure 3 shows PPI images of the polarimetric variables observed by the MP-X radar at 0159:54 LST when 
(a) $Z_{H}$-uncor $(d B Z)$

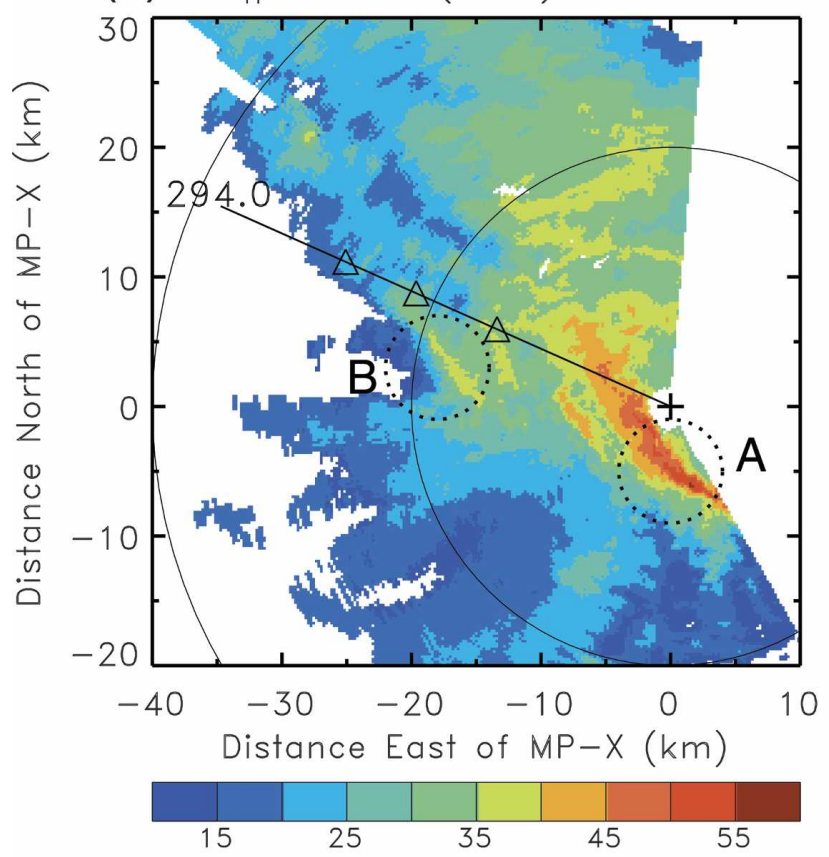

(c) $K_{D P}\left(\right.$ deg $\left.\mathrm{km}^{-1}\right)$

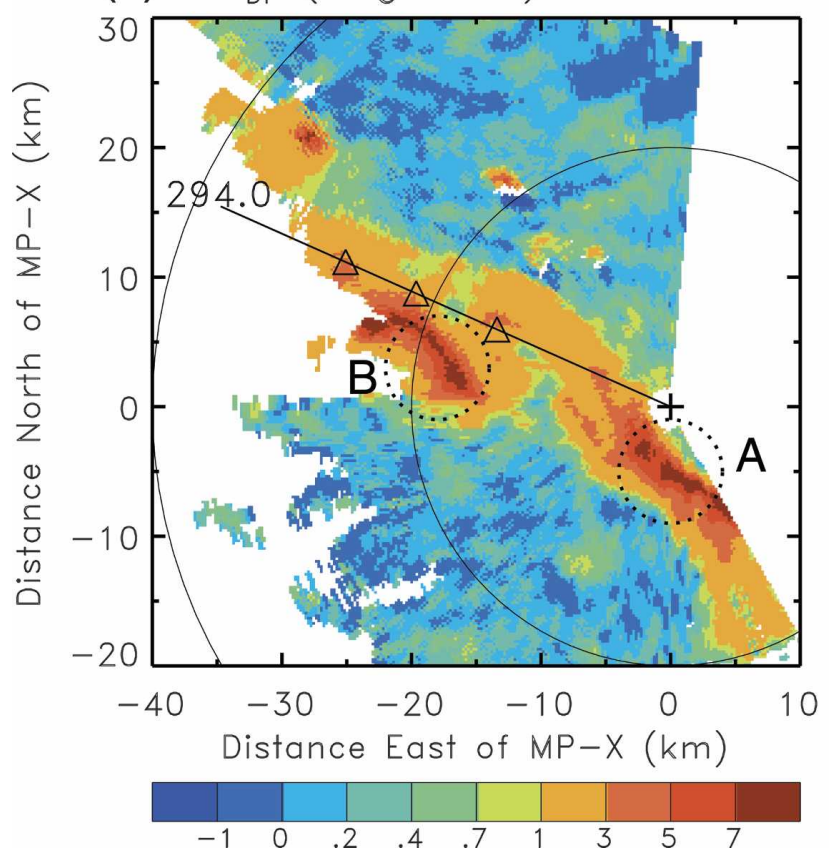

(b) $Z_{D R}-$ uncor $(d B)$

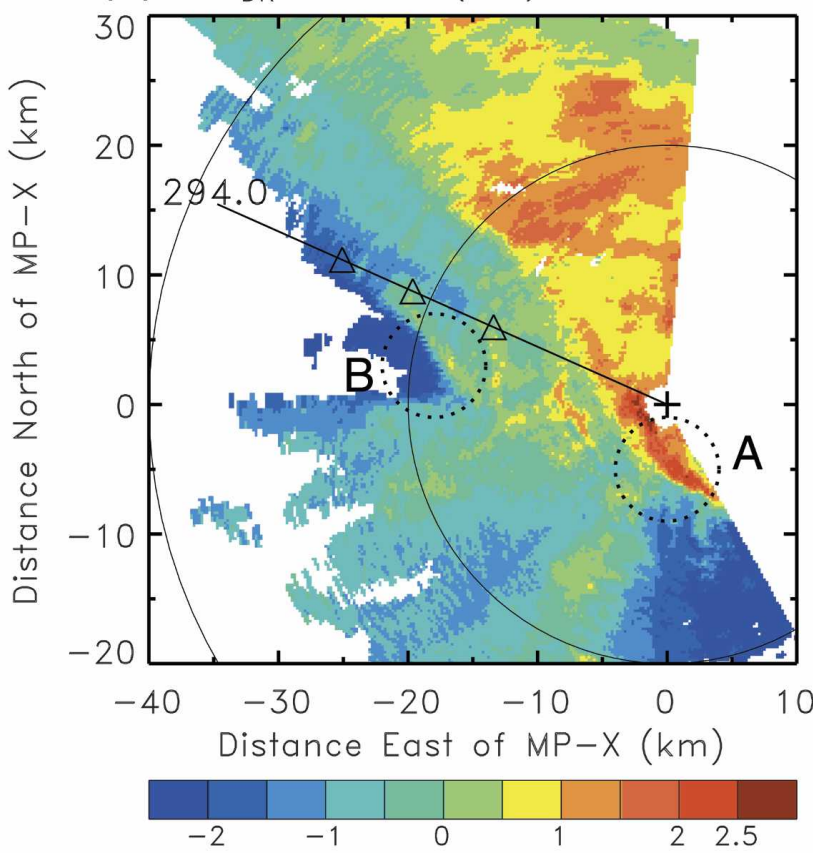

(d)

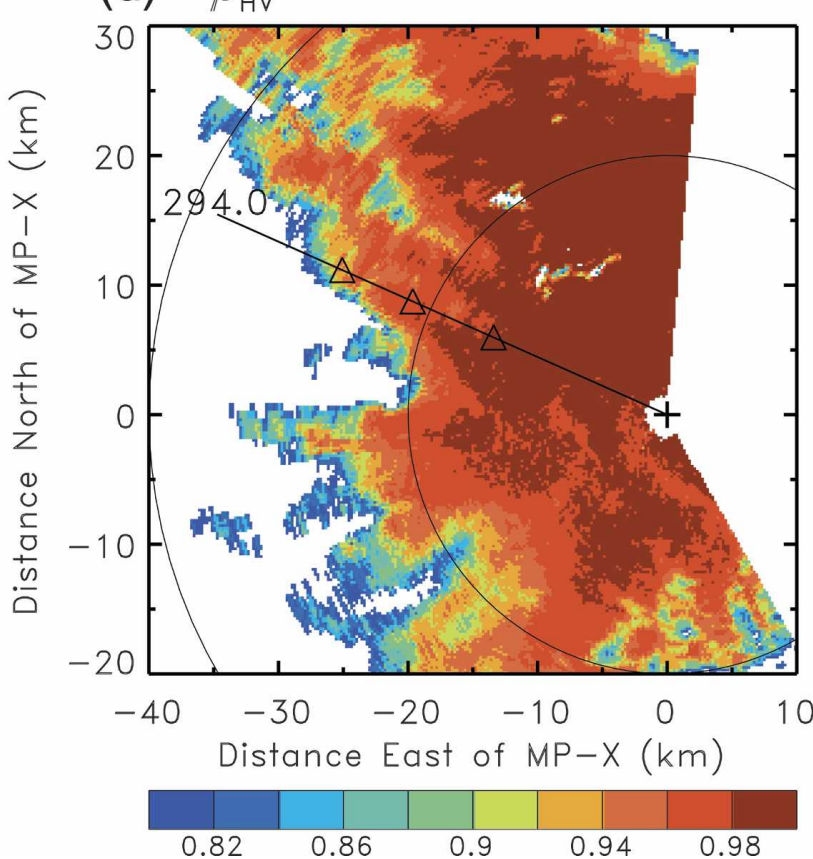

FIG. 3. PPI images of polarimetric variables observed at an elevation angle of $2.5^{\circ}$, at 0159:54 LST 11 Sep 2001: (a) $Z_{\mathrm{H}}$, (b) $Z_{\mathrm{DR}}$, (c) $K_{\mathrm{DP}}$, and (d) $\rho_{\mathrm{HV}}$. The $\Delta$ marks denote the locations of the disdrometers.

severe attenuation occurred. A strong "line"-type echo occurred in region A adjacent to the radar site (Fig. 3a), with a high reflectivity factor over $50 \mathrm{dBZ}$. Behind this strong echo, the measured radar reflectivity factor decreases with increasing distance from the radar. The pattern of $Z_{\mathrm{DR}}$ also shows a decrease with increasing distance (Fig. $3 b$ ). In particular, $Z_{\mathrm{DR}}$ values are negative in the region behind the strong echo A. Meanwhile, in the northern area where the radar beam does not propagate through the strong echo $\mathrm{A}$, the $Z_{\mathrm{DR}}$ image 

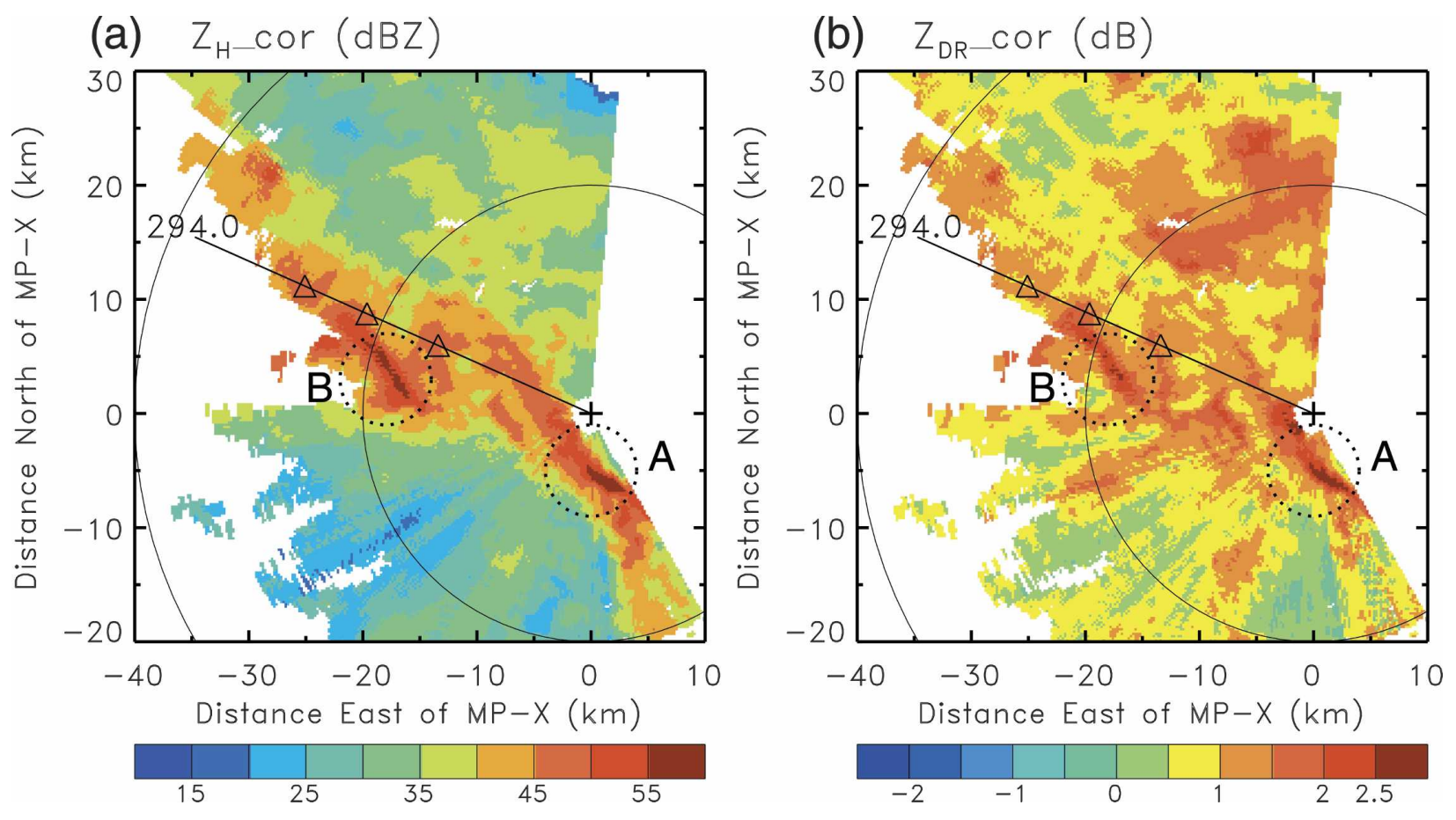

FIG. 4. Same as Fig. 3, except for (a) $Z_{\mathrm{H}}$ and (b) $Z_{\mathrm{DR}}$ corrected for rain attenuation.

shows large and positive values. In the $K_{\mathrm{DP}}$ image (Fig. $3 c$ ), a strong echo with high $K_{\mathrm{DP}}$ above $7^{\circ} \mathrm{km}^{-1}$ occurred in region A where high reflectivity was also observed. The $K_{\mathrm{DP}}$ image shows another strong echo in region $\mathrm{B}$ at ranges from 15 to $25 \mathrm{~km}$ along an azimuth angle of $294^{\circ}$. Note that the $K_{\mathrm{DP}}$ values near echo B are similar to those found in echo A. However, the echo B is not well recognized in the measured $Z_{\mathrm{H}}$ and $Z_{\mathrm{DR}}$ images, where the reflectivity factor and differential reflectivity are below $40 \mathrm{~dB} Z$ and $1 \mathrm{~dB}$, respectively, and are much smaller than those in echo A. These differences between the $K_{\mathrm{DP}}$ image and the measured $Z_{\mathrm{H}}$ and $Z_{\mathrm{DR}}$ images indicate that the western area experienced severe attenuation because of the strong echo A near the radar site.

Note that the data in Fig. 3 are on the PPI surface of $2.5^{\circ}$ elevation angle, whose corresponding heights are about 1.7 (at 40-km range) and 3.5 (at 80-km range) km, which are lower than the height of the melting level (about $4 \mathrm{~km}$ ). In addition, $Z_{\mathrm{DR}}$ generally increases as $Z_{\mathrm{H}}$ increases, and the $\rho_{\mathrm{HV}}$ pattern (Fig. 3d) displays high values (above 0.9 ) over most regions. The decrease of $\rho_{\mathrm{HV}}$ values below 0.9 with increasing distance from the radar is the result of a decrease of SNR caused by severe attenuation.

Figure 4 shows corrected $Z_{\mathrm{H}}$ and $Z_{\mathrm{DR}}$ images obtained from the correction algorithm for rain attenuation. Compared to the patterns in Fig. 3, the weak re- flectivity below $40 \mathrm{~dB} Z$ in region B shown in Fig. 3a has now been increased up to $55 \mathrm{~dB} Z$, owing to the attenuation correction. These corrected $Z_{\mathrm{H}}$ values in region $\mathrm{B}$ are now similar to those in region $\mathrm{A}$, and are consistent with the $K_{\mathrm{DP}}$ pattern in the two regions. The measured negative $Z_{\mathrm{DR}}$ values in Fig. 3b have also been corrected to large and positive values. Thus, the patterns of $Z_{\mathrm{H}}$ and $Z_{\mathrm{DR}}$ after correction are consistent with the $K_{\mathrm{DP}}$ pattern (Fig. 3c), which is not affected by attenuation.

\section{b. Comparison with simulated values based on disdrometer data}

To validate the corrected $Z_{\mathrm{H}}$ and $Z_{\mathrm{DR}}$ values shown in Fig. 4, we compare them with simulated values using drop spectra measured by three ground-based disdrometers located along the $294^{\circ}$ azimuth angle at ranges of $14.7,21.5$, and $27.5 \mathrm{~km}$ marked with $\Delta$. Figure 5 shows the polarimetric variables along the beam at the azimuth angle of $294^{\circ}$. Also shown in Fig. 5 are the results of scattering simulations at the three ranges denoting the averaged values based on the disdrometer DSDs using the three relations for drop shapes and the temperature of $15^{\circ} \mathrm{C}$. The error bars denote maximum and minimum values of the simulations for the three different relations for drop shapes described in Part I. Note that the "minimum" relation for drop shapes corresponds to the maximum values of the error bar, because it produces the largest oblateness, while the Keenan 

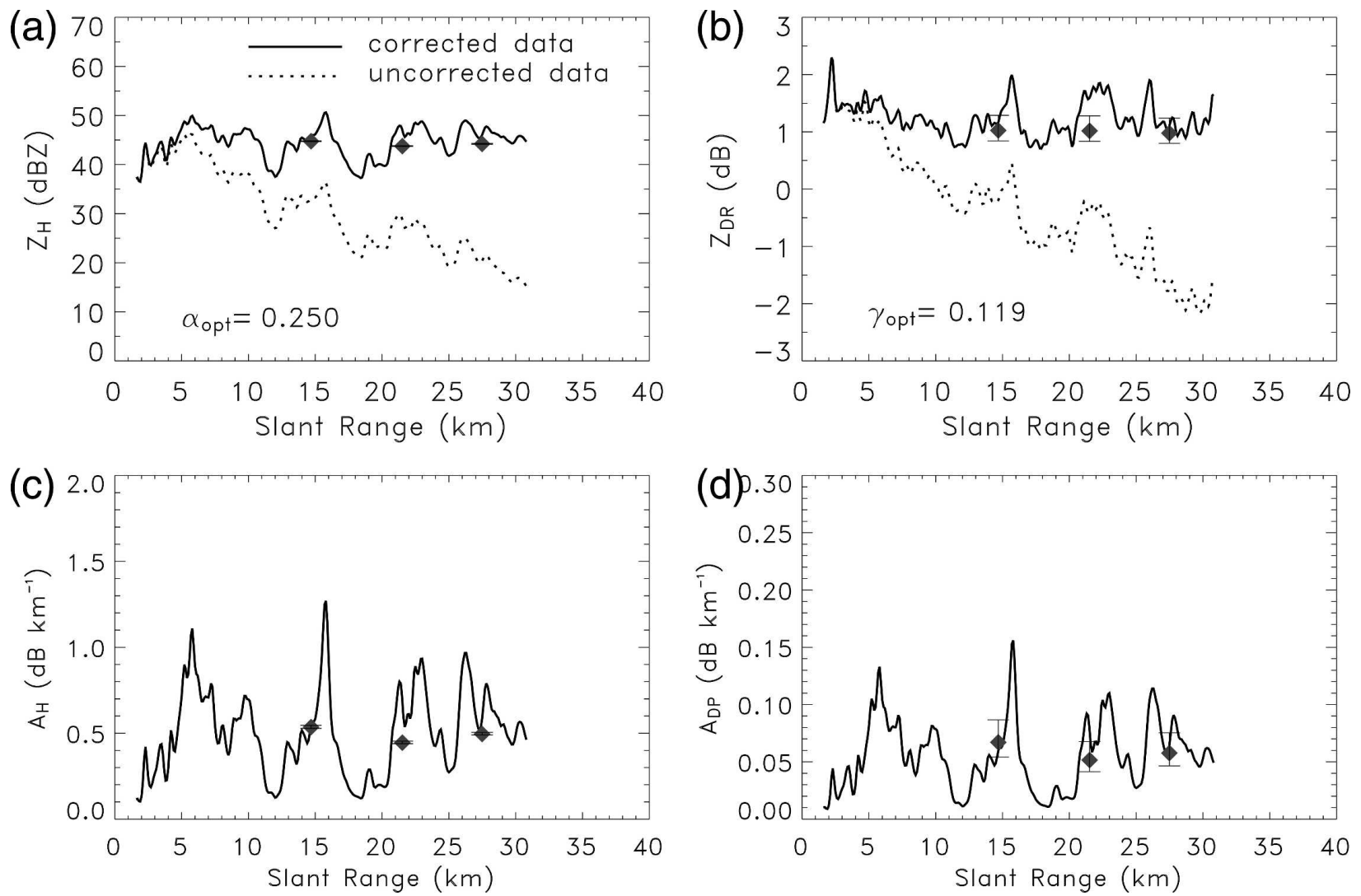

FIG. 5. Range profiles of polarimetric variables along an azimuth angle of $294^{\circ}$ at 0159:54 LST 11 Sep 2001: (a) $Z_{\mathrm{H}}$, (b) $Z_{\mathrm{DR}}$, (c) $A_{\mathrm{H}}$, and (d) $A_{\mathrm{DP}}$. The $\diamond$ marks denote the mean values from the simulations based on disdrometer data for the three relations for drop shapes and the temperature of $15^{\circ} \mathrm{C}$. The error bars denote maximum and minimum values for the three different relations for drop shapes.

relation corresponds to the minimum values, because of the smallest oblateness. The simulated values using disdrometer DSD data were further averaged for $3 \mathrm{~min}$, because the PPI scan was repeated about every $3 \mathrm{~min}$, while the disdrometer data were 1-min averaged. As shown in the figure, the $Z_{\mathrm{H}}$ and $Z_{\mathrm{DR}}$ values uncorrected for attenuation (Figs. 5a and 5b) show large differences from the simulated values based on the disdrometer data. Note that, as expected, the $Z_{\mathrm{H}}$ and $Z_{\mathrm{DR}}$ values before attenuation correction gradually decrease with increasing range from the radar. In particular, negative $Z_{\mathrm{DR}}$ values occur at ranges beyond about $10 \mathrm{~km}$. In contrast to the uncorrected data, the corrected $Z_{\mathrm{H}}$ and $Z_{\mathrm{DR}}$ values agree well with the simulated data. At the range of $30 \mathrm{~km}$, the $Z_{\mathrm{H}}$ value was increased from 16 to $45 \mathrm{~dB} Z$ and the $Z_{\mathrm{DR}}$ value was increased from -2 to 1 $\mathrm{dB}$, owing to the attenuation correction. Before the attenuation correction, the $Z_{\mathrm{H}}$ and $Z_{\mathrm{DR}}$ values measured by the radar at the three disdrometer sites have averaged absolute deviations of 17.0 and $1.79 \mathrm{~dB}$, respectively, defined as an averaged value of absolute deviations between the radar data and the mean simulated data (i.e., the $\diamond$ marks) at the three disdrometer sites, where the radar data were averaged over a range interval of $0.3 \mathrm{~km}$. On the other hand, these deviations decrease significantly in the corrected $Z_{\mathrm{H}}$ and $Z_{\mathrm{DR}}$ values, with the averaged absolute deviations of 1.8 and 0.26 $\mathrm{dB}$, respectively, which are comparable to their respective measurement errors.

The range profiles of $A_{\mathrm{H}}$ and $A_{\mathrm{DP}}$ (Figs. 5c and 5d), which were derived in the correction procedure, also show quite good agreement with the simulated values. The averaged absolute deviations of $A_{\mathrm{H}}$ and $A_{\mathrm{DP}}$ are 0.11 and $0.014 \mathrm{~dB} \mathrm{~km}^{-1}$, respectively. Because these attenuation profiles were used in the procedure for correction of $Z_{\mathrm{H}}$ and $Z_{\mathrm{DR}}$ attenuation, the good agreement of the attenuation profiles shown in the figure validates to some degree the modified attenuation-correction methodology. In Fig. 5, another notable result may be that the simulated $Z_{\mathrm{DR}}$ and $A_{\mathrm{DP}}$ values present large variations with drop shape, that is, large deviations bounded by the maximum and minimum values, while the $Z_{\mathrm{H}}$ and $A_{\mathrm{H}}$ values present relatively small deviations. The simulated $Z_{\mathrm{H}}$ values have only $0.2 \mathrm{~dB}$ as an 
averaged deviation for three disdrometers with a mean value of about $44 \mathrm{dBZ}$, while the averaged deviation of $Z_{\mathrm{DR}}$ reaches $0.4 \mathrm{~dB}$ with a mean value of about $1 \mathrm{~dB}$. Similarly, the deviation of $A_{\mathrm{H}}\left(0.02^{\circ} \mathrm{km}^{-1}\right)$ is smaller than that of $A_{\mathrm{DP}}\left(0.03^{\circ} \mathrm{km}^{-1}\right)$. This is because $Z_{\mathrm{H}}$ and $A_{\mathrm{H}}$ are less sensitive to drop shape.

The profiles of $A_{\mathrm{H}}$ and $A_{\mathrm{DP}}$ in Fig. 5 correspond to $\alpha$ and $\gamma$ of $0.250 \mathrm{~dB}\left({ }^{\circ}\right)^{-1}$ and 0.119 , respectively. These are the optimal values determined in the correction algorithm as described in section $2 \mathrm{~b}$ of Part I. Figure $6 a$ shows a comparison of the error defined by (8) of Part I, for the same beam as in Fig. 5, between the measured $\phi_{\mathrm{DP}}$ profile (filtered by the FIR scheme) and that derived from several $\alpha$ values within the range from 0.025 to $0.575 \mathrm{~dB}\left({ }^{\circ}\right)^{-1}$. As shown in the figure, the error term is minimal at the $\alpha$ value of 0.250 . The $\phi_{\mathrm{DP}}$ profile derived from this optimal $\alpha$ is in excellent agreement with the measured (and filtered) $\phi_{\mathrm{DP}}$ profile (Fig. 6b). On the contrary, large differences from the filtered $\phi_{\mathrm{DP}}$ profile arise when the $\alpha$ values of 0.15 and $0.35 \mathrm{~dB}\left({ }^{\circ}\right)^{-1}$ are assumed as an optimal value. The averaged absolute deviations of $Z_{\mathrm{H}}, Z_{\mathrm{DR}}, A_{\mathrm{H}}$, and $A_{\mathrm{DP}}$ when the $\alpha$ value of 0.15 was assumed are $2.6 \mathrm{~dB}, 0.49 \mathrm{~dB}, 0.20 \mathrm{~dB}$ $\mathrm{km}^{-1}$, and $0.018 \mathrm{~dB} \mathrm{~km}^{-1}$, respectively. The deviations of $Z_{\mathrm{H}}$ and $Z_{\mathrm{DR}}$ correspond to roughly 3 and 2 times their measurement errors, respectively. In this case, the optimal $\gamma$ is automatically set to 0.175 and the $Z_{\mathrm{H}}$ $\left(Z_{\mathrm{DR}}\right)$ range profile presents undercorrection (overcorrection) (not shown here), because the decrease of $\alpha$ results in increasing $\gamma$ as in (11) of Part I. When the $\alpha$ value of 0.35 was assumed as an optimal value, the averaged absolute deviations of $Z_{\mathrm{H}}, Z_{\mathrm{DR}}, A_{\mathrm{H}}$, and $A_{\mathrm{DP}}$ are $3.0 \mathrm{~dB}, 0.19 \mathrm{~dB}, 0.25 \mathrm{~dB} \mathrm{~km}^{-1}$, and $0.023 \mathrm{~dB} \mathrm{~km}^{-1}$, respectively. In this case, the optimal $\gamma$ is determined as 0.095 , which results in a decrease of $Z_{\mathrm{DR}}$ and increase of $Z_{\mathrm{H}}$. Comparing the averaged absolute deviations with the $\alpha$ and $\gamma$ values, those deviations for the optimal $\alpha\left[0.250 \mathrm{~dB}\left({ }^{\circ}\right)^{-1}\right]$ and $\gamma(0.119)$ described above are relatively small, though the deviation of $Z_{\mathrm{DR}}$ for $\alpha=$ 0.35 (corresponding to $\gamma=0.095$ ) is somewhat smaller than that for the optimal $\alpha$ and $\gamma$. This result supports the validity of the determination procedure of the optimal $\alpha$ and $\gamma$ and, further, the corrected $Z_{\mathrm{H}}$ and $Z_{\mathrm{DR}}$ values by the modified methodology for the X-band radar described in this study.

In the $Z_{\mathrm{DR}}$ correction procedure, the constraint $Z_{\mathrm{DR}}\left(r_{0}\right)$ is determined from the corrected $Z_{\mathrm{H}}$ values basically based on the $Z_{\mathrm{DR}}-Z_{\mathrm{H}}$ relation (1). Therefore, the correction of $Z_{\mathrm{DR}}$ may be affected by an inherent error in mean $Z_{\mathrm{DR}}-Z_{\mathrm{H}}$ relation and an error embedded in the corrected $Z_{\mathrm{H}}$ value. For the error embedded in the corrected $Z_{\mathrm{H}}$ value, it does not have a significant
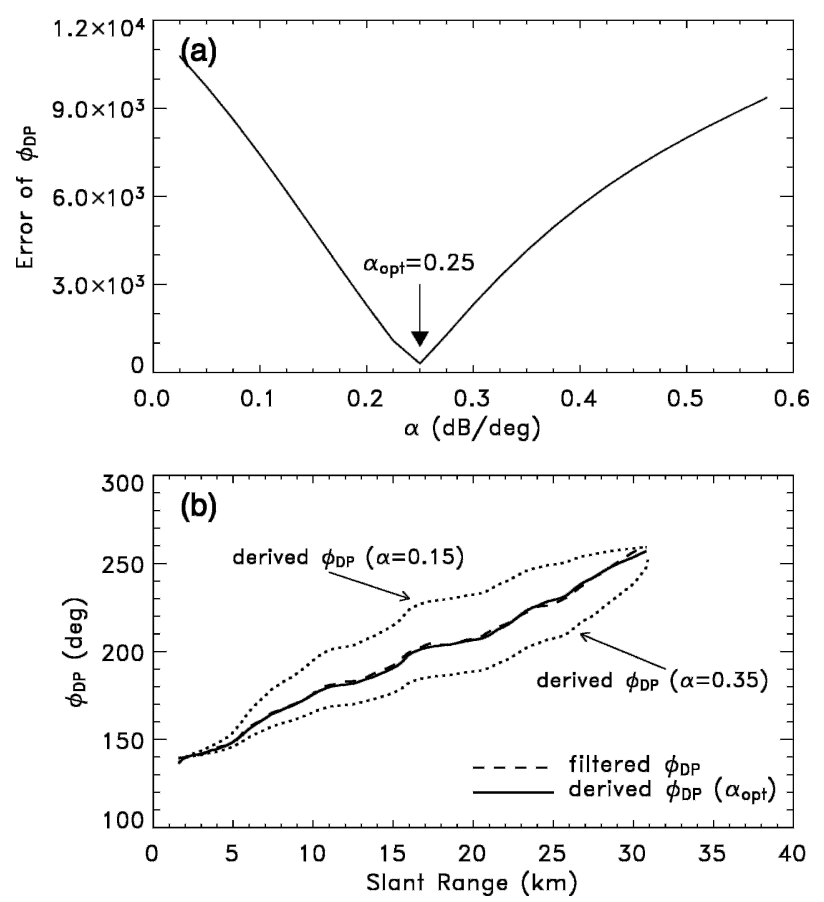

FIG. 6. (a) Error of $\phi_{\mathrm{DP}}$ between the filtered values and the derived values [see Eq. (8)] vs the $\alpha$ value, at the same azimuth angle as in Fig. 5. (b) Comparison of the filtered $\phi_{\mathrm{DP}}$ and the derived $\phi_{\mathrm{DP}}$ from the optimal $\alpha$ value $\left[0.250 \mathrm{~dB}\left({ }^{\circ}\right)^{-1}\right]$.

influence on the $Z_{\mathrm{DR}}$ correction, because an error in $Z_{\mathrm{H}}$ results in a $Z_{D R}$ variation corresponding to the amount multiplied by the slope parameter $(0.051)$ in the $Z_{\mathrm{DR}^{-}}$ $Z_{\mathrm{H}}$ relation (1). For example, the error of about $2 \mathrm{~dB}$ embedded in the corrected $Z_{\mathrm{H}}$ range profile shown in Fig. 5 causes a $Z_{\mathrm{DR}}$ variation of only $0.1 \mathrm{~dB}$, which is smaller than the $Z_{\mathrm{DR}}$ measurement error $(0.25 \mathrm{~dB})$. However, an error in the mean $Z_{\mathrm{DR}}-Z_{\mathrm{H}}$ relation (1) may be an important factor for obtaining reliable corrected $Z_{\mathrm{DR}}$ values. To analyze this effect, we examined variations of the $Z_{\mathrm{DR}}$ range profiles when \pm 0.25 and $\pm 0.5 \mathrm{~dB}$ were added in the original $Z_{\mathrm{DR}}\left(r_{0}\right)$ value $(1.44$ $\mathrm{dB}$ ) for the $Z_{\mathrm{DR}}$ profile shown in Fig. 5. Herein, the error $0.5 \mathrm{~dB}$ corresponds roughly to the standard deviation of $Z_{\mathrm{DR}}$ to be estimated by the $Z_{\mathrm{DR}}-Z_{\mathrm{H}}$ relation (see Fig. 5 of Part I) and, further, 2 times the $Z_{\mathrm{DR}}$ measurement error. When $+0.25(-0.25) \mathrm{dB}$ was added as an error in the $Z_{\mathrm{DR}}\left(r_{0}\right)$ constraint, the averaged absolute deviations of $Z_{\mathrm{DR}}$ and $A_{\mathrm{DP}}$ were 0.42 (0.20) $\mathrm{dB}$ and $0.016(0.013) \mathrm{dB} \mathrm{km}^{-1}$, respectively. When $+0.50(-0.50) \mathrm{dB}$ was added, the averaged absolute deviations of $Z_{\mathrm{DR}}$ and $A_{\mathrm{DP}}$ were $0.58(0.24 \mathrm{~dB})$ and $0.018(0.014) \mathrm{dB} \mathrm{km}^{-1}$, respectively. Thus, the accuracy of the $Z_{\mathrm{DR}}$ correction heavily depends on accuracy of the $Z_{\mathrm{DR}}\left(r_{0}\right)$ constraint. A better relationship than the mean $Z_{\mathrm{DR}}-Z_{\mathrm{H}}$ relation (1) used in this study 

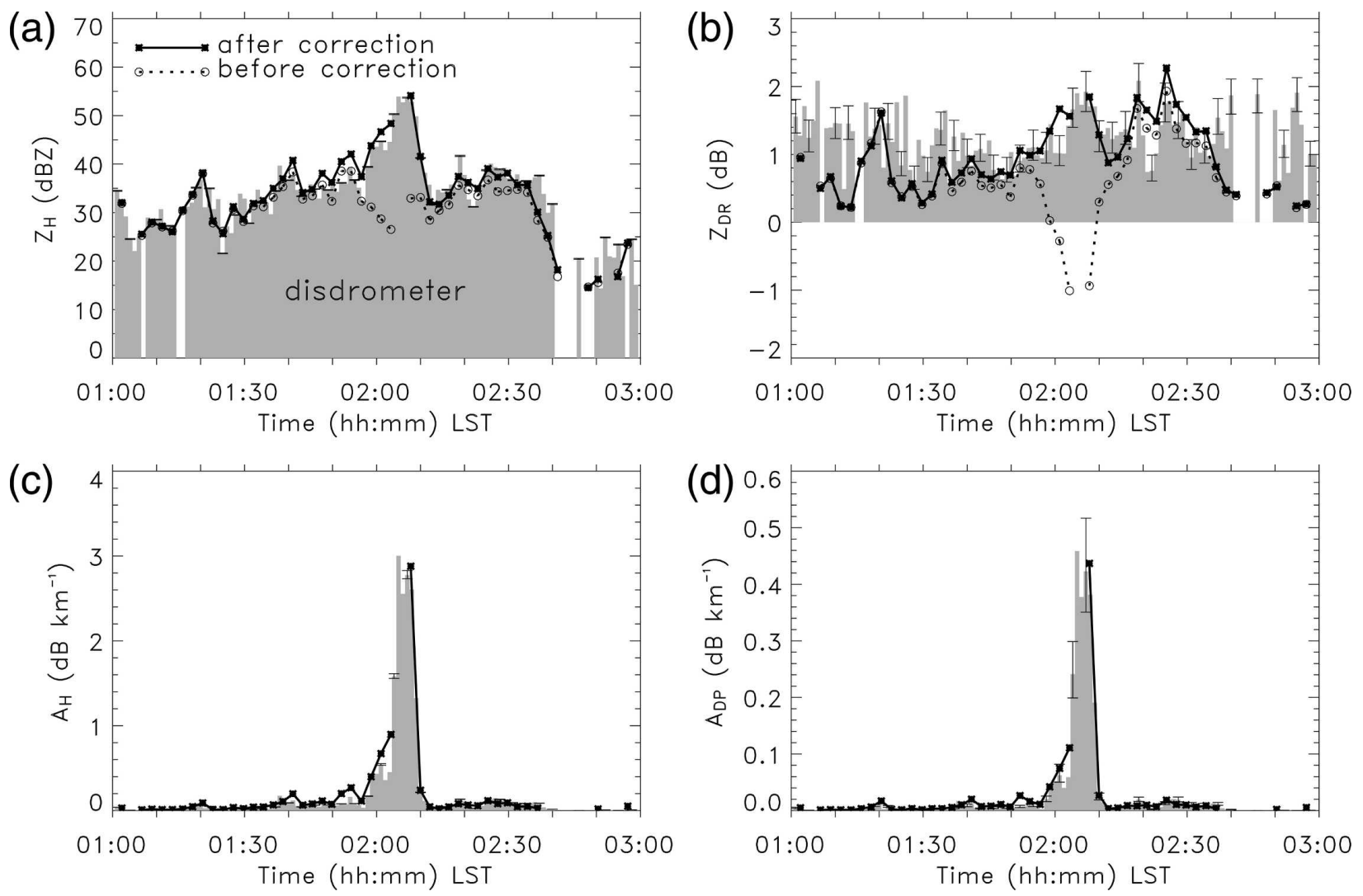

FIG. 7. Comparisons of the polarimetric variables (a) $Z_{\mathrm{H}}$, (b) $Z_{\mathrm{DR}}$, (c) $A_{\mathrm{H}}$, and (d) $A_{\mathrm{DP}}$ over the disdrometer site located at the range of $21.5 \mathrm{~km}$, from 0100 to 0300 LST 11 Sep 2001. The gray shaded area and the error bar denote the simulated values based on the disdrometer data under the same conditions as in Fig. 5.

may be necessary for improving the accuracy of the $Z_{\mathrm{DR}}$ correction.

Figure 7 shows comparisons of temporal variations of the polarimetric variables over the disdrometer site located at the range of $21.5 \mathrm{~km}$. To compare the two different datasets, the radar data were averaged over five ranges (i.e., range sector of $0.5 \mathrm{~km}$ ) and three consecutive azimuth rays (about $1.5^{\circ}$ ) centered over the disdrometer site, on the PPI surface at the elevation angle of $2.5^{\circ}$. The simulated values using disdrometer data were averaged for $3 \mathrm{~min}$, because the PPI scan was repeated about every $3 \mathrm{~min}$. The averaged simulated values are denoted by the gray shading and the error bars overlapping in the gray shaded area denote the maximum and minimum values corresponding to the "minimum" and Keenan relations for drop shapes, respectively. Figure 7 a shows that the uncorrected $Z_{\mathrm{H}}$ values are significantly lower than the simulated $Z_{\mathrm{H}}$ values, in particular, near 0200 LST when the strong rainband shown in Fig. 4 passed over the disdrometer site. The uncorrected $Z_{\mathrm{H}}$ value at $0204 \mathrm{LST}$ is about 22 $\mathrm{dB} Z$, but the simulated value reaches $53 \mathrm{~dB} Z$. In contrast, the corrected $Z_{\mathrm{H}}$ values show quite good agree- ment with the simulated values over the entire period. The uncorrected $Z_{\mathrm{DR}}$ values are also very low (about $-1 \mathrm{~dB}$ ) near 0200 LST and show large differences from the simulated values, while after correction the $Z_{\mathrm{DR}}$ values are more consistent with the simulated $Z_{\mathrm{DR}}$. Figures $7 \mathrm{c}$ and $7 \mathrm{~d}$ show the $A_{\mathrm{H}}$ and $A_{\mathrm{DP}}$ values derived in the correction procedure, respectively. Accompanying the passage of the strong rainband, peaks of $A_{\mathrm{H}}$ and $A_{\mathrm{DP}}$ occur near $0200 \mathrm{LST}$, with maximum values of about 3.0 and $0.46 \mathrm{~dB} \mathrm{~km}^{-1}$, respectively. The variations of $A_{\mathrm{H}}$ and $A_{\mathrm{DP}}$ with time are in good agreement with the simulations based on disdrometer data.

In Fig. $7 \mathrm{~b}$, although the corrected $Z_{\mathrm{DR}}$ values near 0200 LST agree well with the simulated values, large differences appear near 0110 LST and from 0240 to 0300 LST. It appears that these differences in $Z_{\mathrm{DR}}$ values may be ascribed to the difficulty of comparing a volume-averaged radar measurement with point disdrometer measurements, rather than to errors in the attenuation correction procedure itself. For example, at 0112 LST the increase of $\phi_{\mathrm{DP}}$ from the radar to the disdrometer location is very small (below $5^{\circ}$; not shown here). Under this circumstance, it is difficult to expect 
that differential attenuation resulted in the large decrease of $Z_{\mathrm{DR}}$ values of about $1 \mathrm{~dB}$ as seen in Fig. $7 \mathrm{~b}$. Rather than being an effect of attenuation, therefore, it may be the result of sample volume differences between the radar and disdrometer along with DSD gradients within the larger radar sample volume. Note that the simulated $Z_{\mathrm{DR}}$ value based on disdrometer DSD data near 0110 LST is similar to the value near 0200 LST, when the corrected $Z_{\mathrm{DR}}$ well agreed with the simulated value, but the $Z_{\mathrm{H}}$ values near 0110 LST are much smaller compared to those at 0200 LST (by about $20 \mathrm{~dB}$ ). Assuming a radar sampling volume with high variation of DSDs, this result may be explained by the hypothesis that the simulated $Z_{\mathrm{DR}}$ values from disdrometer data were affected by a few large drops whose concentration cannot be estimated accurately, while the radar $Z_{\mathrm{DR}}$ values were lower because of DSD gradients within the larger radar sampling volume. Such gradients are more likely to occur at the beginning and end times of the passage of line echo B (in Fig. 3c, e.g.) over the disdrometer site at a range of $21.5 \mathrm{~km}$. In conclusion, the temporal comparisons shown in Fig. 7 between radar-corrected $Z_{\mathrm{H}}$ and $Z_{\mathrm{DR}}$ values (as well as the radar-estimated $A_{\mathrm{H}}$ and $A_{\mathrm{DP}}$ values) with corresponding disdrometer-based scattering simulations further demonstrate the accuracy of the attenuation-correction methodology described in this study.

\section{c. Internal comparison using the characteristics among the polarimetric variables}

For a more "global" validation of the correction algorithm, we compare the corrected $Z_{\mathrm{H}}$ and $Z_{\mathrm{DR}}$ values (also $A_{\mathrm{H}}$ and $A_{\mathrm{DP}}$ ) with empirical relations among the polarimetric variables obtained from scattering simulations based on disdrometer data. Figure 8 shows the comparisons of $K_{\mathrm{DP}}$ versus $Z_{\mathrm{H}}, A_{\mathrm{H}}$ versus $Z_{\mathrm{H}}$, and $Z_{\mathrm{DR}}$ versus $Z_{\mathrm{H}}$. The comparisons with the uncorrected $Z_{\mathrm{H}}$ and $Z_{\mathrm{DR}}$ values are presented in the left panels, while the right panels are for the corrected values. The radar data points in the figure are from each resolution volume on the PPI surface at the elevation angle of $2.5^{\circ}$ and, hence, they have various heights from the surface to about $3.5 \mathrm{~km}$, which implies different temperatures. To consider the temperature variation with height, the scattering simulations using ground-based disdrometer data were performed for the three different temperatures $\left(0^{\circ}, 15^{\circ}\right.$, and $\left.30^{\circ} \mathrm{C}\right)$. For drop shapes, the three different relations were considered. In Fig. 8, the upper dotted lines correspond to simulations at the temperature of $0^{\circ} \mathrm{C}$ and the minimum relation for drop shapes, while the lower dotted lines correspond to the temperature of $30^{\circ} \mathrm{C}$ and the Keenan relation. It is shown in Fig. 8a that, for a given $K_{\mathrm{DP}}$, the uncorrected $Z_{\mathrm{H}}$ values are much smaller than those expected from the simulated $K_{\mathrm{DP}}-Z_{\mathrm{H}}$ relations. Similar patterns are also observed in the comparisons of $A_{\mathrm{H}}$ versus $Z_{\mathrm{H}}$ (Fig. 8c) and $Z_{\mathrm{DR}}$ versus $Z_{\mathrm{H}}$ (Fig. 8e). After correction, on the contrary, the corrected $Z_{\mathrm{H}}$ and $Z_{\mathrm{DR}}$ values lie in the range predicted by the scattering simulations, as shown in the right panels. It is worthwhile to note that the $A_{\mathrm{H}}-Z_{\mathrm{H}}$ relations in Figs. $8 \mathrm{c}$ and $8 \mathrm{~d}$ are not sensitive to temperature and drop shape, as mentioned in section 4 of Part I.

Figure 9 shows similar comparisons between radarderived and simulations of $A_{\mathrm{H}}$ versus $K_{\mathrm{DP}}$ and $A_{\mathrm{DP}}$ versus $A_{\mathrm{H}}$. The upper and lower dotted lines in the $A_{\mathrm{H}}-K_{\mathrm{DP}}$ comparison (Fig. 9a) have the opposite meaning to that in Fig. 8 (i.e., the upper dotted line corresponds to the temperature of $30^{\circ} \mathrm{C}$ and the Keenan relation, while the lower line is for the temperature of $0^{\circ} \mathrm{C}$ and the minimum relation), while those lines in the $A_{\mathrm{DP}}-A_{\mathrm{H}}$ comparison (Fig. 9b) have the same meaning as in Fig. 8. As shown in Fig. 9a, the radar data scatterplot of $A_{\mathrm{H}}$ versus $K_{\mathrm{DP}}$ lay in the range predicted from the simulations. Between $A_{\mathrm{DP}}$ and $A_{\mathrm{H}}$ (Fig. 9b), a similar good agreement is also shown. Thus, the functional dependence of radar polarimetric variables after correction for attenuation agrees well with those based on scattering simulations and provide an additional measure of validation of the attenuation-correction methodology.

Figure 10 shows scatterplots of the coefficient $\alpha$ of the $A_{\mathrm{H}^{-}}-K_{\mathrm{DP}}$ relation and the coefficient $\gamma$ of the $A_{\mathrm{DP}^{-}}$ $A_{\mathrm{H}}$ relation. These coefficients are the optimal values determined in the correction procedure. As shown in the figure, the optimal $\alpha$ varies widely from 0.05 to 0.55 $\mathrm{dB}\left({ }^{\circ}\right)^{-1}$. Most $(73 \%)$ of the optimal $\alpha$ values, however, lie in the range from 0.139 to 0.335 , which is the range obtained from the scattering simulations in Part I. The modal value of optimal $\alpha$ occurs at 0.275 , which is close to the mean value obtained from the scattering simulations (0.254). The modal value of optimal $\gamma$ occurs near 0.135 , which is also close to the mean value from the scattering simulations (0.139). Further, the pairs of $\alpha$ and $\gamma$ obtained from the radar measurements are compared with those from the scattering simulations, in order to test consistency between $\alpha$ and $\gamma$, which have an inversely proportional relationship because the $A_{\mathrm{DP}^{-}}$ $A_{\mathrm{H}}$ relation, which is derived from the $A_{\mathrm{H}}-K_{\mathrm{DP}}$ and $A_{\mathrm{DP}}-K_{\mathrm{DP}}$ relations with a power-law form. As shown in the figure, the pairs from the radar measurements (plus signs) and from the scattering simulations (black dots) for three temperatures and three drop shape relations reveal the trend that $\gamma$ decreases with increasing $\alpha$. 
(a)

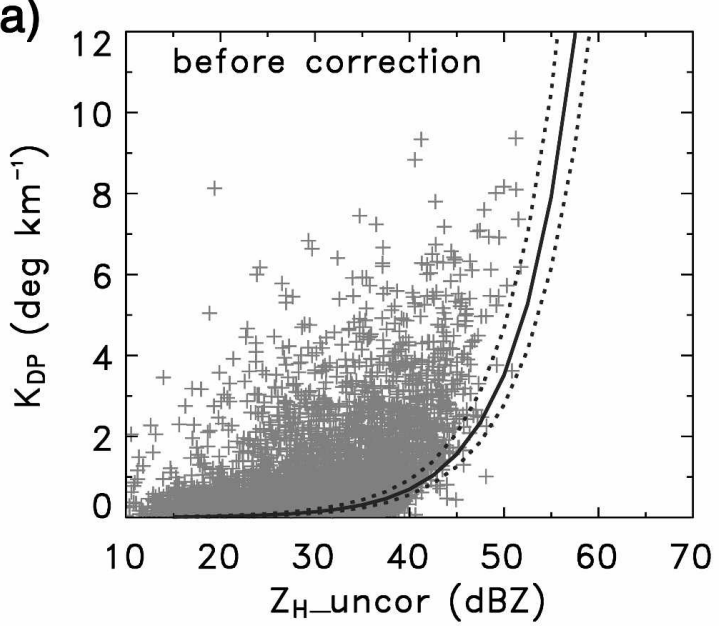

(c)

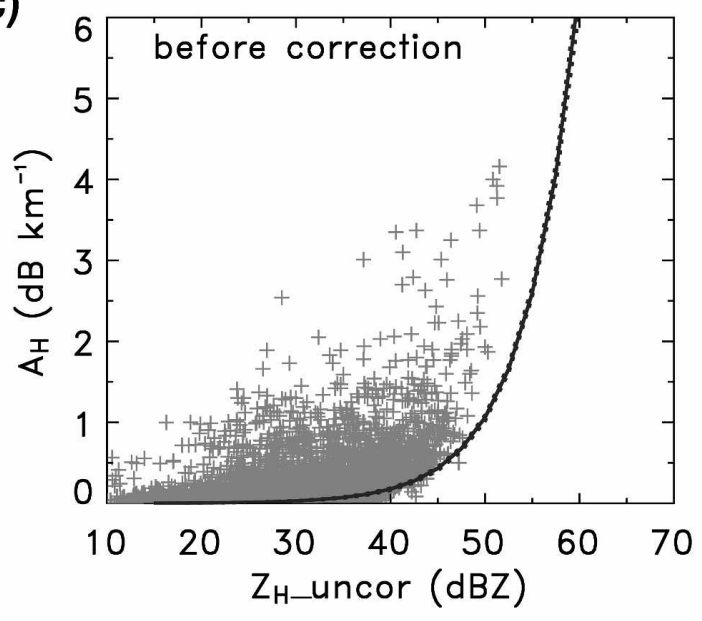

(e)

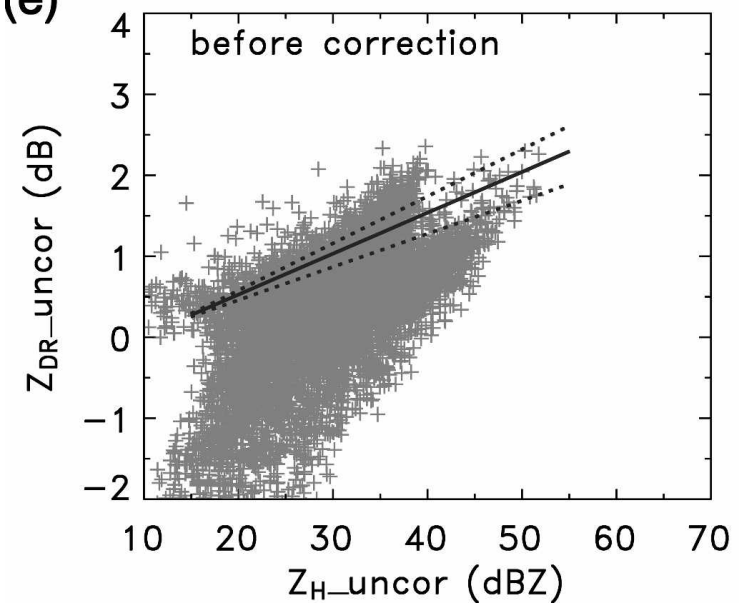

(b)

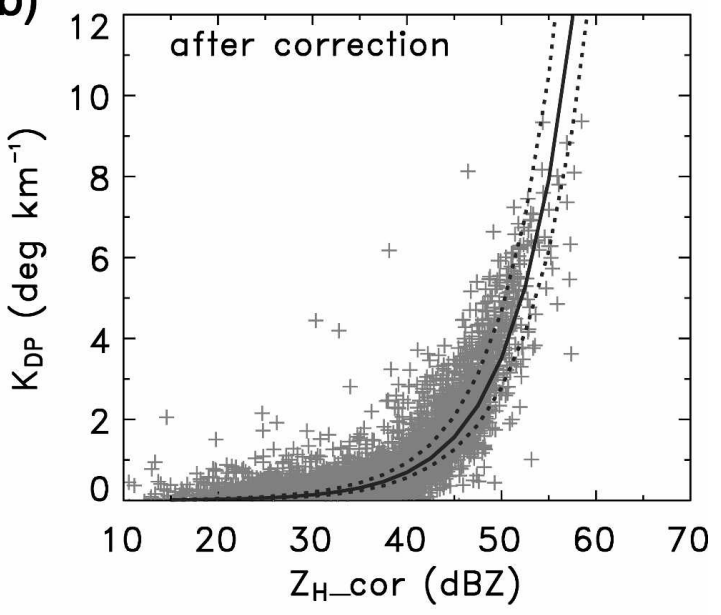

(d)

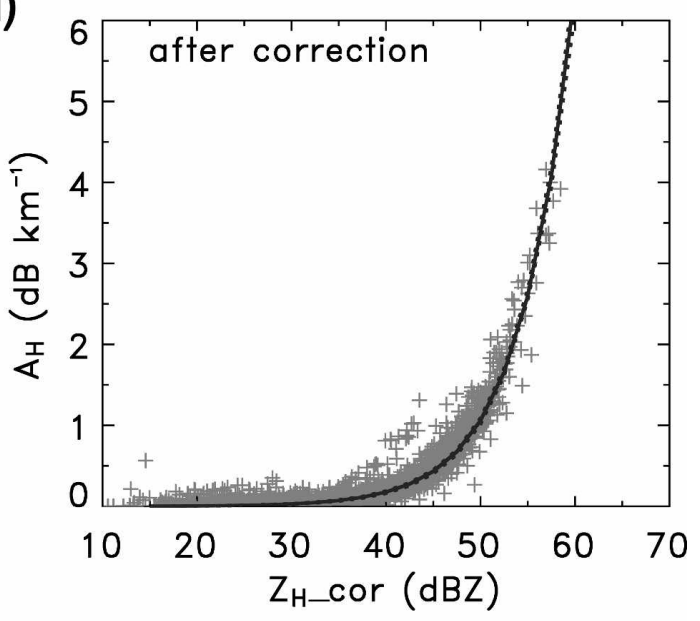

(f)

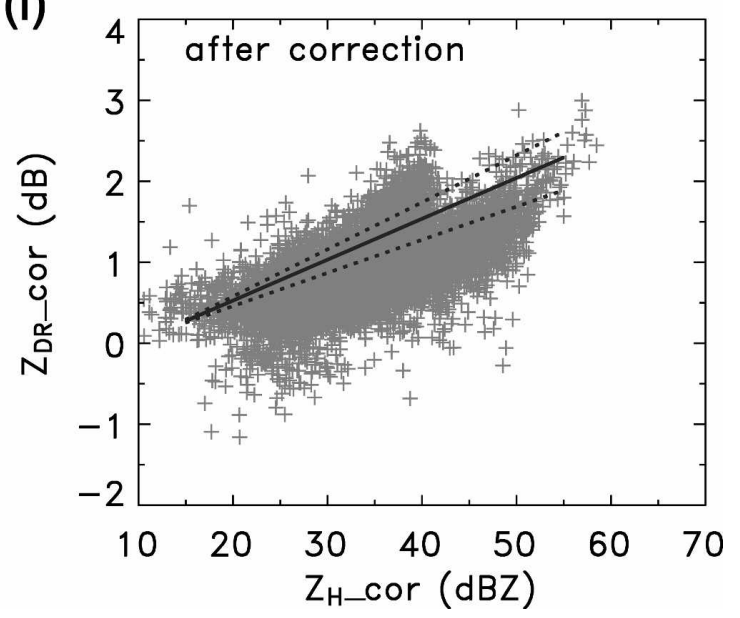

FIG. 8. Scatterplots of $K_{\mathrm{DP}}$ vs $Z_{\mathrm{H}}, A_{\mathrm{H}}$ vs $Z_{\mathrm{H}}$, and $Z_{\mathrm{DR}}$ vs $Z_{\mathrm{H}}$ from 0100 to 0300 LST 11 Sep 2001: (a), (c), and (e) comparisons with the uncorrected $Z_{\mathrm{H}}$ and $Z_{\mathrm{DR}}$, and (b), (d), and (f) the corrected data for attenuation. The solid and dashed lines are empirical relations derived from scattering simulations for three different temperatures and three different drop shape relations. The sold line corresponds to an averaged relation and the dotted lines correspond to maximum and minimum values (or, upper and lower bound) among the relations for each condition. 
(a)

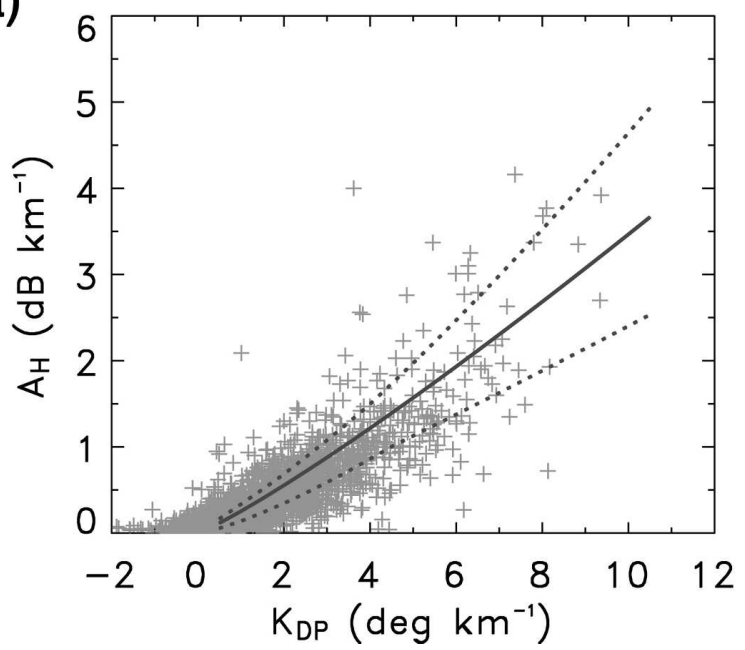

(b)

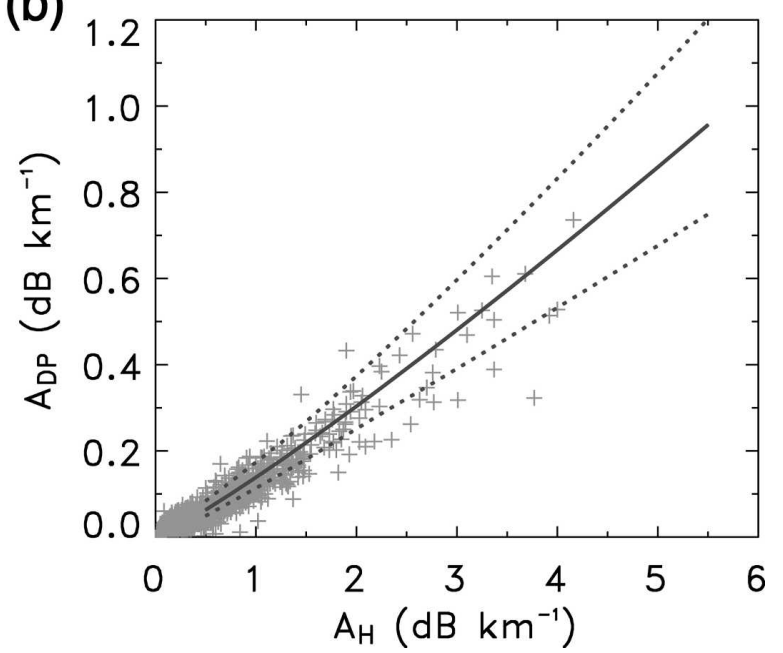

FIg. 9. Same as Fig. 8, except for (a) $A_{\mathrm{H}}$ vs $K_{\mathrm{DP}}$ and (b) $A_{\mathrm{DP}}$ vs $A_{\mathrm{H}}$.

Moreover, the pairs of $\alpha$ and $\gamma$ obtained from the scattering simulations occur within the region of a relatively high frequency of occurrence of the optimal $\alpha$ and $\gamma$ values (contours) determined from the radar measurements, though three points are obvious outliers, with $\alpha$ values below $0.2 \mathrm{~dB}\left({ }^{\circ}\right)^{-1}$. Although the three points with $\alpha$ values below $0.2 \mathrm{~dB}\left({ }^{\circ}\right)^{-1}$ present an ill consistency, they are the values corresponding to the minimum drop shape relation. As will be shown later, the $K_{\mathrm{DP}}$-based rainfall estimates show better agreement with gauge measurements when the Andsager and Keenan drop shape relations are used, rather than the minimum relation. This result means that most of the radar measurements used in this study are better described by the Andsager and Keenan relations than by the minimum relation. Thus, the optimal $\alpha$ and $\gamma$ derived from radar measurements are consistent with those obtained from scattering simulations. This result again supports the accuracy of the attenuationcorrection methodology used in this study.

\section{Estimation of rainfall amounts and raindrop size distributions}

\section{a. Estimation of rainfall amounts}

To examine the effect of attenuation on rainfall estimation using the MP-X radar, in the present study two radar-rainfall estimators were considered as follows:

$$
R\left(Z_{\mathrm{H}}\right)=\left\{\begin{array}{lll}
7.07 \times 10^{-3} Z_{\mathrm{H}}^{0.819} & \text { for } & Z_{\mathrm{H}} \leq 35 \mathrm{dBZ} \\
7.40 \times 10^{-2} Z_{\mathrm{H}}^{0.566} & \text { for } & Z_{\mathrm{H}}>35 \mathrm{dBZ}
\end{array}\right.
$$

$$
\begin{aligned}
& R\left(K_{\mathrm{DP}}\right) \\
& \quad= \begin{cases}R\left(Z_{\mathrm{H}} \leq 35 \mathrm{dBZ}\right) & \text { for } K_{\mathrm{DP}} \leq 0.3^{\circ} \mathrm{km}^{-1} \\
& \text { or } Z_{\mathrm{H}} \leq 35 \mathrm{dBZ} \\
19.63 K_{\mathrm{DP}}^{0.823} & \text { otherwise }\end{cases}
\end{aligned}
$$

(where $R, Z_{\mathrm{H}}$, and $K_{\mathrm{DP}}$ are in $\mathrm{mm} \mathrm{h}^{-1}, \mathrm{~mm}^{6} \mathrm{~m}^{-3}$, and ${ }^{\circ} \mathrm{km}^{-1}$, respectively). The radar-rainfall estimators

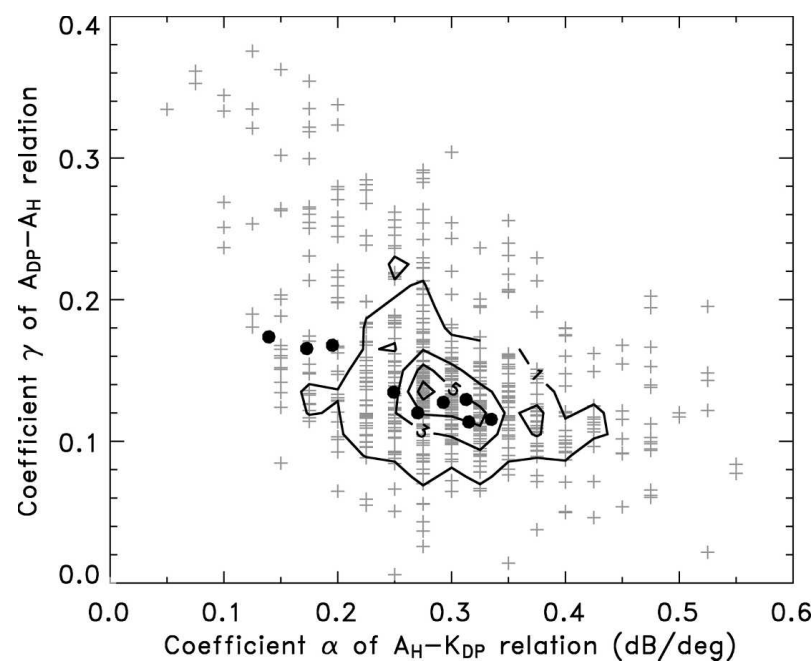

FIG. 10. Scatterplots of the coefficients of the $A_{\mathrm{H}}-K_{\mathrm{DP}}$ and $A_{\mathrm{DP}}-A_{\mathrm{H}}$ relations. The plus signs denote the optimal $\alpha$ and $\gamma$ determined from the radar measurements during 0100-0300 LST 11 Sep 2001, and their relative frequency of occurrence is denoted by contours with an interval of $2 \%$ starting from $1 \%$. The black dots denote the coefficients of the relations derived from scattering simulations for three temperatures and three drop shape relations. 
above were derived from scattering simulations using the same drop spectra as described in section 3 of Part I, under the conditions of temperature of $15^{\circ} \mathrm{C}$ and the Andsager relation for drop shapes. Nonlinear regression analysis was employed in derivation of the estimators. The $R\left(Z_{\mathrm{H}}\right)$ estimator was divided into stratiform and convective types, with a threshold of $35 \mathrm{~dB} Z$. The two $Z-R$ relations in (2a) are representative of stratiform $\left(Z=422 R^{1.22}\right)$ and maritime convective $(Z=$ $\left.100 R^{1.77}\right)$ rain types. The $R\left(K_{\mathrm{DP}}\right)$ estimator is a composite one with a threshold of $0.3^{\circ} \mathrm{km}^{-1}$, which is the estimated standard error of $K_{\mathrm{DP}}$. For cases with $K_{\mathrm{DP}}<$ $0.3^{\circ} \mathrm{km}^{-1}$ or $Z_{\mathrm{H}}<35 \mathrm{dBZ}$, the stratiform $R\left(Z_{\mathrm{H}}\right)$ estimate is used.

Figure 11 shows PPI images of rainfall rates estimated from the MP-X radar data at an elevation angle of $2.5^{\circ}$ and at 0159 LST 11 September 2001, which is the same time as that in Fig. 3. It is shown that the pattern of $R\left(Z_{\mathrm{H}_{-}}\right.$uncor $)$estimates from the uncorrected $Z_{\mathrm{H}}$ (Fig. 11a) is different from both the $R\left(Z_{\mathrm{H}_{-}}\right.$cor) estimates from the corrected $Z_{\mathrm{H}}$ (Fig. 11b) and the composite $R\left(K_{\mathrm{DP}}\right)$ estimates with $R\left(Z_{\mathrm{H}_{-}}\right.$cor $)$estimates (Fig. 11c). The composite $R\left(K_{\mathrm{DP}}\right)$ estimates show the two rain echoes of line type in regions $\mathrm{A}$ and $\mathrm{B}$, where the rainfall rates are similar to each other, and are higher than $64 \mathrm{~mm} \mathrm{~h}^{-1}$. However, the $R\left(Z_{\mathrm{H}_{-}}\right.$uncor $)$ estimates show much weaker rainfall rates, as expected, over the whole region. In particular, the rainfall rates within the echo $\mathrm{B}$ region are less than $16 \mathrm{~mm} \mathrm{~h}^{-1}$. This underestimation of $R\left(Z_{\mathrm{H}_{-}}\right.$uncor $)$estimates is a result of the severe attenuation caused by the strong echo A, as shown in Fig. 3. In contrast to $R\left(Z_{\mathrm{H}_{-}}\right.$uncor $)$, the $R\left(Z_{\mathrm{H}_{-}}\right.$cor $)$estimates shows a similar distribution to the $R\left(K_{\mathrm{DP}}\right)$ estimates. The high rainfall rates above $64 \mathrm{~mm}$ $\mathrm{h}^{-1}$ occurs at both A and B echoes, though the widths in the radial direction of the echoes are wider in the $R\left(K_{\mathrm{DP}}\right)$ estimates than in the $R\left(Z_{\mathrm{H}_{-}}\right.$cor $)$estimates, because $K_{\mathrm{DP}}$ is calculated after filtering the $\phi_{\mathrm{DP}}$ in the range, whereas the $Z_{\mathrm{H}}$ measurement is available at each range resolution volume. Figure 12 shows a comparison of radar estimates with gauge measurements over the disdrometer site located at the range of 21.5 $\mathrm{km}$. In this figure, the $R\left(K_{\mathrm{DP}}\right)$ and $R\left(Z_{\mathrm{H}_{-}}\right.$cor $)$estimates present well the high rainfall rates measured by the disdrometer around $0205 \mathrm{LST}$, while the $R\left(Z_{\mathrm{H}-}\right.$ uncor $)$ estimates, as expected, cannot estimate the peak values. In the figure, the gap in the radar estimates near 0205 LST is a result of the very weak returned signal, which is caused by a decrease of SNR by severe attenuation, as mentioned earlier.

In 2003 the MP-X radar was moved to Ebina (near Tokyo) for a field project to study the operational ap- plication of X-band radar for very heavy rainfall events in mountainous and flat metropolitan topography. Three long-duration rain events (two stratiform and one typhoon) among the observed data in 2003 were available for comparison with the eight rain gauges, as mentioned in section 2a. Figures 13a and 13b show comparisons of rainfall rates and accumulations measured by gauges and estimated by radar, respectively, for the stratiform event on 15 August 2003. In the figure, the gauge rainfall rates and accumulations are mean values for the second gauge network where four gauges are placed within about a $1 \mathrm{~km}^{2}$ area, about 10 $\mathrm{km}$ away from the radar (Fig. 2). The comparison between the radar estimates and gauge measurements was made by the same space-time averaging method as in Fig. 7 for disdrometer comparisons. As shown in Fig. $13 \mathrm{a}$, the $R\left(Z_{\mathrm{H}-}\right.$ uncor $)$ estimates, as expected, are smaller than the gauge measurements because of attenuation. This underestimation is also obvious in the rainfall accumulation (Fig. 13b), where the underestimation becomes severe with increasing time. Note that the data in Fig. 13 are at an elevation angle of $2.1^{\circ}$, which is not affected by ground clutter, and, hence, an underestimation of $Z_{\mathrm{H}}$ by partial blockage of the radar beam is not expected (Zrnic and Ryzhkov 1996). After correction for attenuation, on the contrary, the $R\left(Z_{\mathrm{H}-}\right.$ cor $)$ estimates have substantially recovered in good agreement with the gauge measurements. However, an underestimation still occurs at high rainfall rates from 0700 to 0800 LST (Fig. 13a). On the other hand, the $R\left(K_{\mathrm{DP}}\right)$ estimates show good agreement with the gauge measurements, in particular, the peaks rainfall rates from 0600 to 0800 LST are in good agreement. Comparing differences of total rainfall accumulations over $15 \mathrm{~h}$ (Fig. 13b), the rain gauges recorded a mean total rainfall amount of $162 \mathrm{~mm}$, while the $R\left(Z_{\mathrm{H} \_}\right.$uncor $)$estimates produced only $114 \mathrm{~mm}$, which indicates an underestimation of $30 \%$. On the other hand, $R\left(Z_{\mathrm{H}_{-}}\right.$cor $)$and $R\left(K_{\mathrm{DP}}\right)$ estimates show good agreement with the variation of the gauge rainfall accumulation, though a slight overestimation was produced after 1100 LST. Over the total duration of $15 \mathrm{~h}$, the $R\left(Z_{\mathrm{H}_{-}}\right.$cor $)$and $R\left(K_{\mathrm{DP}}\right)$ estimates produced a total accumulation of about $173 \mathrm{~mm}$, which corresponds to overestimation of only $6.8 \%$ relative to the gauge measurements. It is worthwhile to note that the second gauge network is only $10 \mathrm{~km}$ from the MP-X radar and the 15 August case shown in Fig. 13 is a stratiform precipitation event. Thus, at $\mathrm{X}$ band the attenuation can cause significant underestimation, even at short ranges and for stratiform rain events.

To further examine the relative accuracy of the ra- 

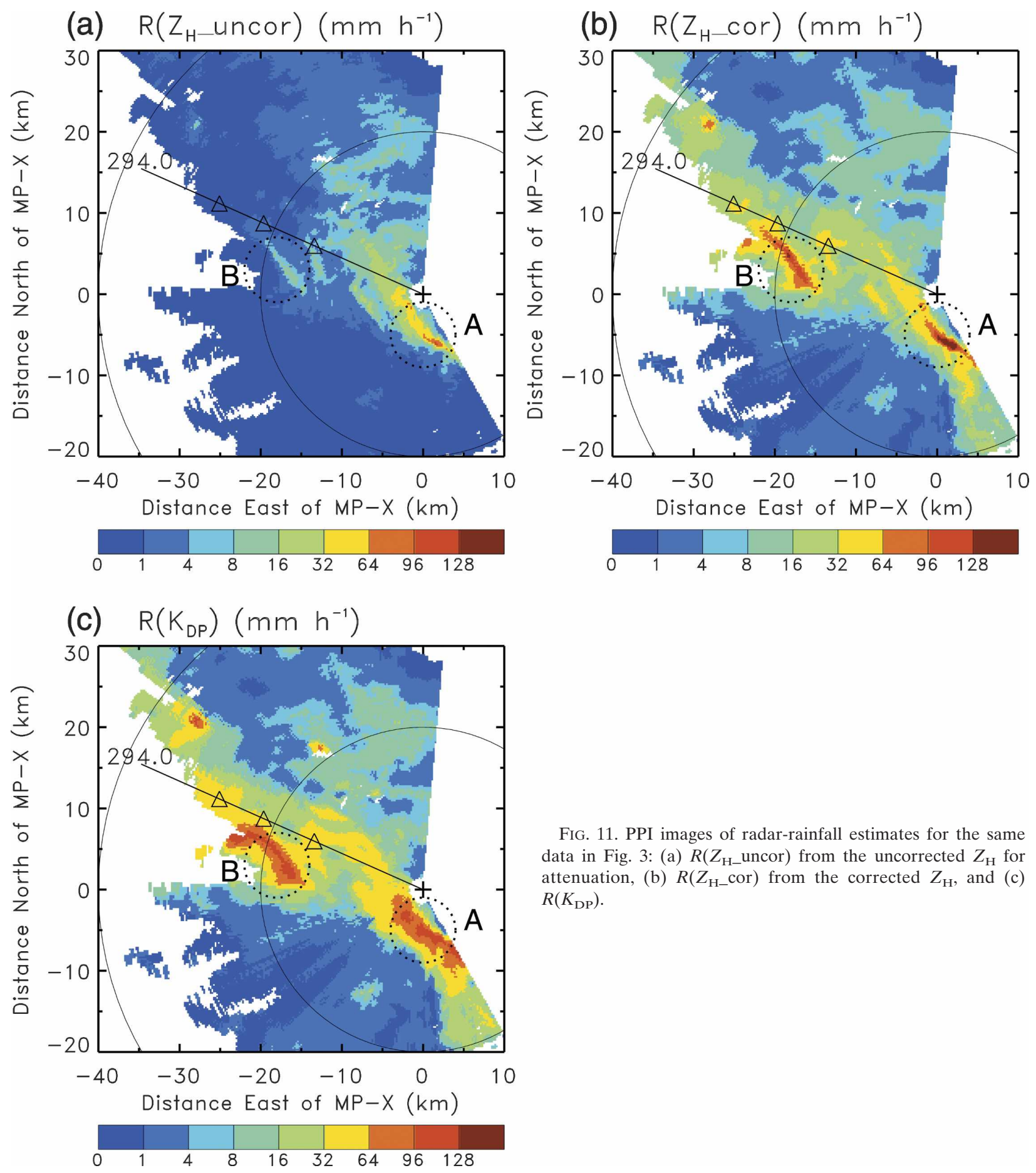

FIG. 11. PPI images of radar-rainfall estimates for the same data in Fig. 3: (a) $R\left(Z_{\mathrm{H}-}\right.$ uncor $)$ from the uncorrected $Z_{\mathrm{H}}$ for attenuation, (b) $R\left(Z_{\mathrm{H}_{-}}\right.$cor) from the corrected $Z_{\mathrm{H}}$, and (c) $R\left(K_{\mathrm{DP}}\right)$.

dar-rainfall estimates, we compare rainfall accumulations over the eight gauges for the three rainfall events. The quantitative comparison of "instantaneous" rainfall rates between radar- and ground-based gauges requires careful attention because of the difference of sampling in time and space (Zawadzki 1975). A useful method for comparison may be the consideration of accumulated rainfall amounts, because the influence resulting from the differences of sampling can be avoided by comparing rainfall accumulations over a time interval and, hence, the effect of attenuation can be retained. However, the comparison of rainfall accumula- 


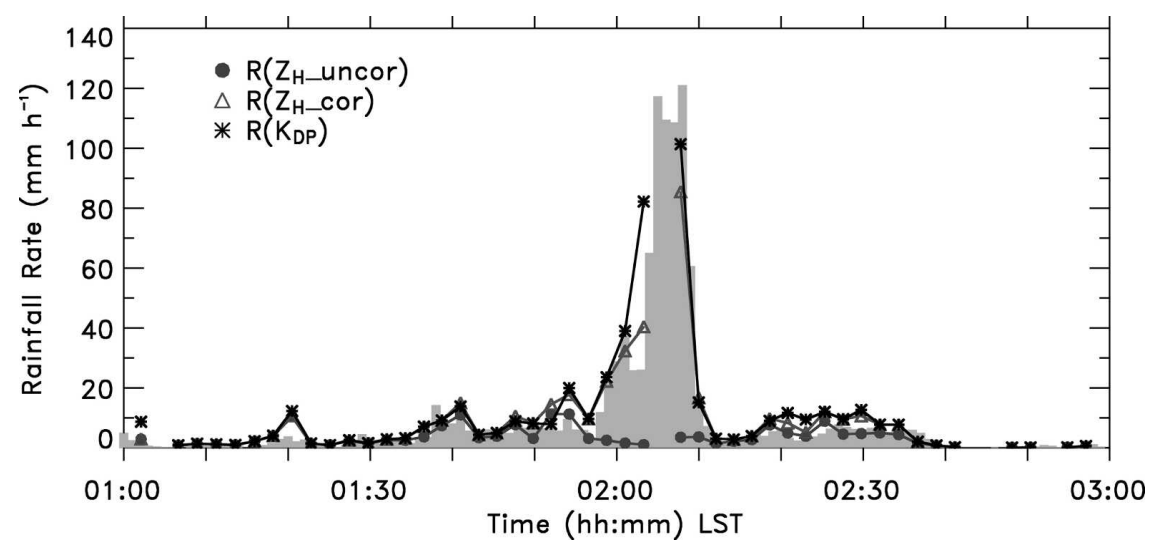

FIG. 12. Comparison of rainfall rates between gauge measurements (gray shaded area) and radar estimates over the disdrometer site located at the range of $21.5 \mathrm{~km}$ from 0100 to 0300 LST 11 Sep 2001.

tions can be affected by the selected time interval. Therefore, in this study the accumulations were considered for three different time intervals of $15 \mathrm{~min}, 1 \mathrm{~h}$, and $3 \mathrm{~h}$, in order to examine dependency of the accu- racy on the time interval. The comparison was quantified by calculating the error of the radar estimates against the gauge measurements, defined by normalized error (NE) and normalized bias (NB) as
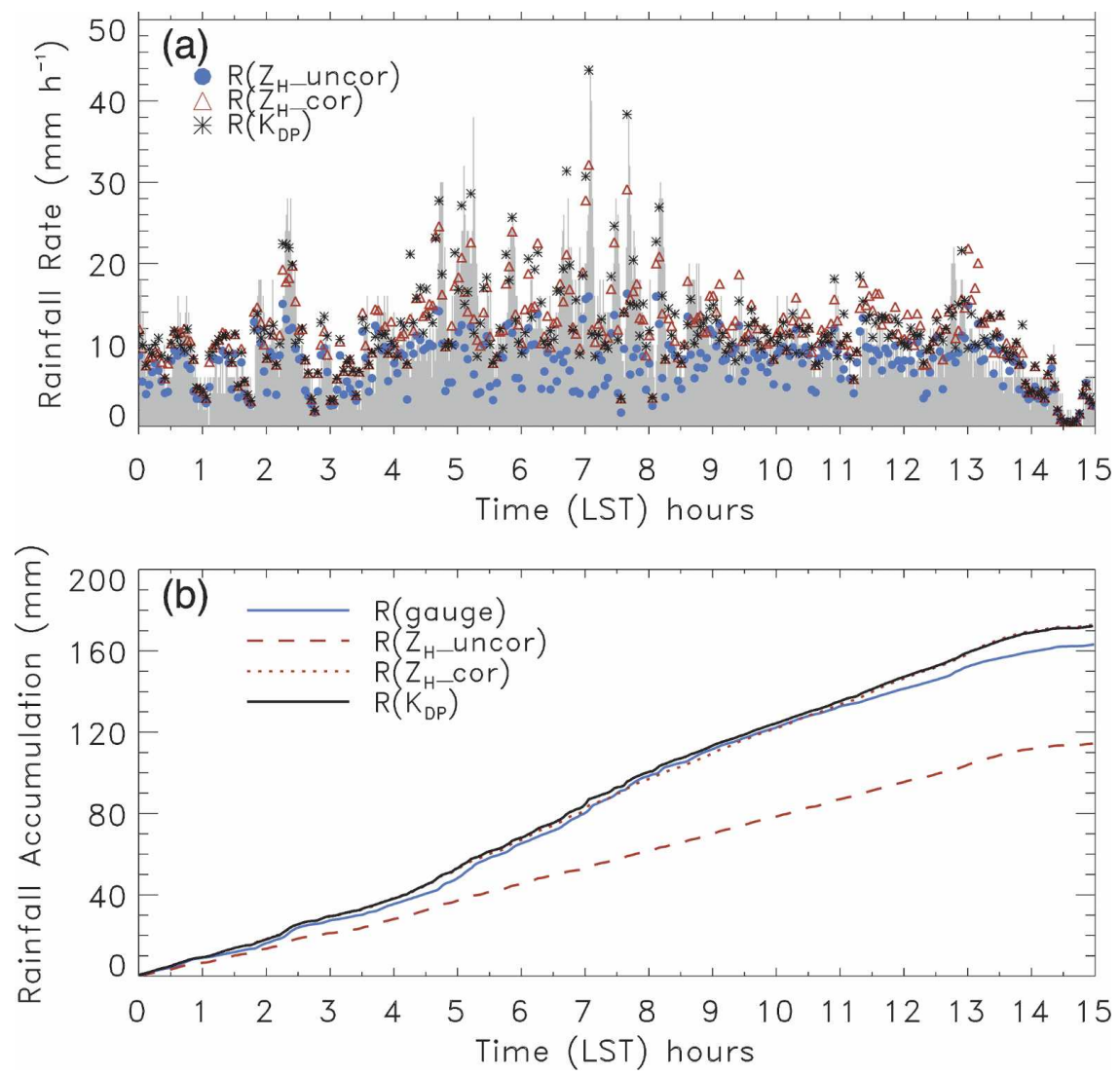

FIG. 13. Comparisons of (a) rainfall rates and (b) accumulations between gauge measurements (gray shaded area) and radar estimates from 0000 to 1500 LST 15 Aug 2003. The gauge measurements are mean values over the second gauge network consisting of four gauges at Hiratsuka (Fig. 2). The radar estimates are at an elevation angle of $2.1^{\circ}$. 

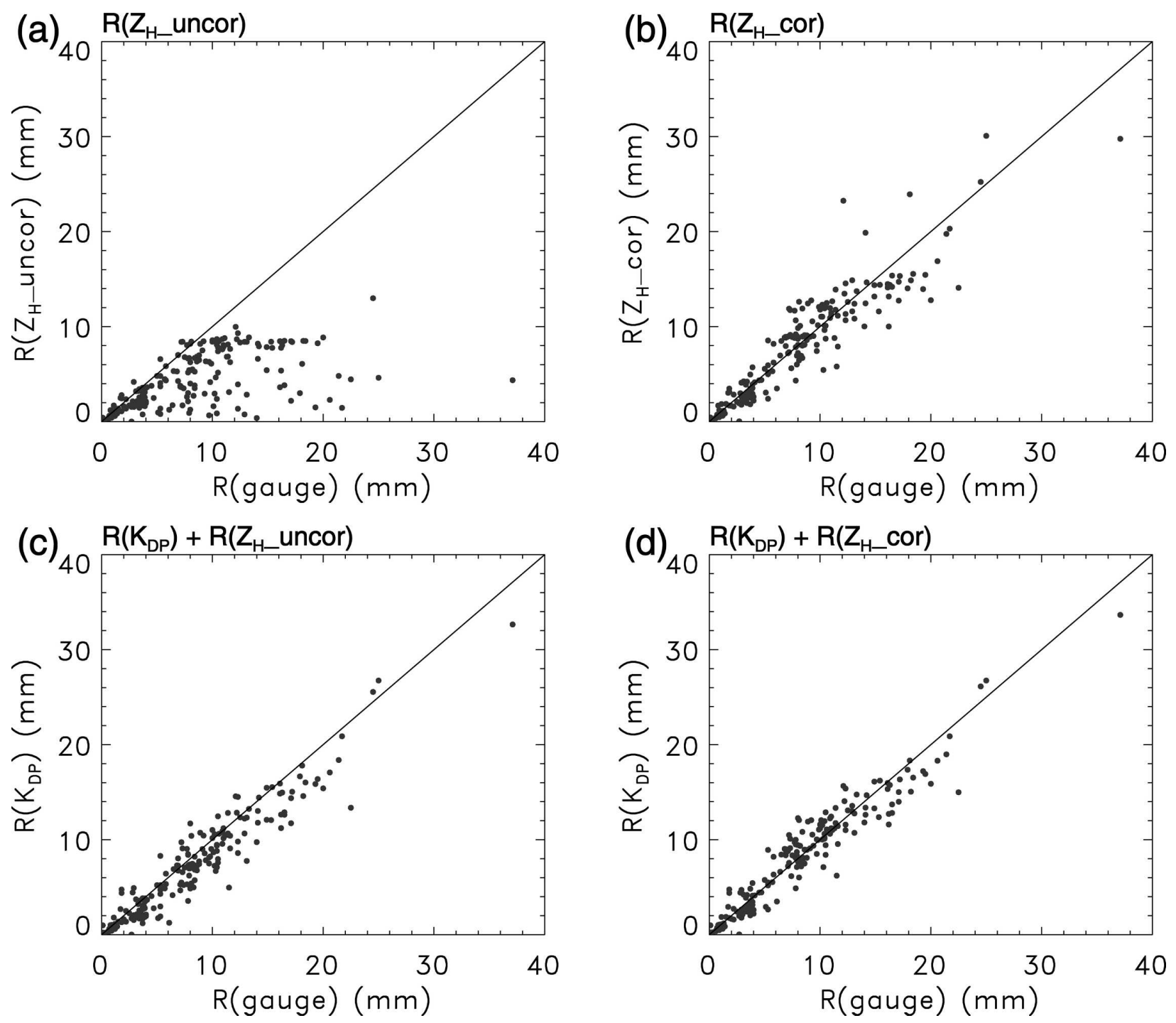

FIG. 14. Comparisons of 1-h rainfall accumulations between gauge measurements and radar estimates for three rain events in 2003: (a) $R\left(Z_{\left.\mathrm{H} \_ \text {uncor }\right)}\right.$ and (b) $R\left(Z_{\mathrm{H}_{-}}\right.$cor $)$. (c), (d) Composite $R\left(K_{\mathrm{DP}}\right)$ estimates with $R\left(Z_{\left.\mathrm{H} \_ \text {uncor}\right)}\right.$ and $R\left(Z_{\mathrm{H} \_}\right.$cor $)$estimates, respectively, when $K_{\mathrm{DP}} \leq 0.3^{\circ} \mathrm{km}^{-1}$ or $Z_{\mathrm{H}-}$ cor $\leq 35 \mathrm{dBZ}$.

$$
\begin{aligned}
\mathrm{NE}= & \left(\frac{1}{N} \sum_{i=1}^{N} \mid R(\text { radar })-R(\text { gauge }) \mid\right) \\
& \left(\frac{1}{N} \sum_{i=1}^{N} R(\text { gauge })\right) \times 100(\%), \\
\mathrm{NB}= & \left(\frac{1}{N} \sum_{i=1}^{N}[R(\text { radar })-R(\text { gauge })]\right) / \\
& \left(\frac{1}{N} \sum_{i=1}^{N} R(\text { gauge })\right) \times 100(\%),
\end{aligned}
$$

where $R$ (radar) and $R$ (gauge) denote radar estimates and gauge measurements, respectively, and $N$ denotes the number of data pairs compared. Figure 14 shows the comparisons of 1-h rainfall accumulation between gauge measurements and radar estimates. Table 2 contains $\mathrm{NE}$ and $\mathrm{NB}$ of the radar-rainfall estimates, for 15-min, 1 - $\mathrm{h}$, and 3-h accumulations. The $R\left(Z_{\mathrm{H}_{-}}\right.$uncor $)$ estimates (Fig. 14a) presents significant underestimation, in particular, the underestimation becomes large with an increasing rainfall amount. The corresponding $\mathrm{NE}$ and $\mathrm{NB}$ are $48.9 \%$ and $-47.6 \%$, respectively (Table 2). After correction for attenuation, on the contrary, the $R\left(Z_{\mathrm{H}-}\right.$ cor) estimates (Fig. $\left.14 \mathrm{~b}\right)$ show good agreement with the gauge measurements. The corresponding NE and NB reduce significantly to $19.1 \%$ and $-2.0 \%$, respectively. Thus, X-band radar reflectivity 
must be corrected for attenuation, for obtaining reliable rainfall amounts using a conventional $Z-R$ relation. However, the precise form of the appropriate $Z-R$ relation to be used will, in general, not be known a priori. It is well-known that an "untuned" $Z-R$ relation can lead to significant bias in rainfall amounts even if the radar is perfectly calibrated and there is no beam blockage. On the contrary, the $R\left(K_{\mathrm{DP}}\right)$ estimator does not depend on radar calibration and is much less sensitive to beam blockage.

Figures $14 \mathrm{c}$ and $14 \mathrm{~d}$ show comparisons with the composite $R\left(K_{\mathrm{DP}}\right)$ estimates, which were superseded by the $R\left(Z_{\mathrm{H} \_}\right.$uncor $)$and $R\left(Z_{\mathrm{H}_{-}}\right.$cor $)$estimates, respectively, when $K_{\mathrm{DP}} \leq 0.3^{\circ} \mathrm{km}^{-1}$ or $Z_{\mathrm{H}-}$ cor $\leq 35 \mathrm{dBZ}$. Compared with the $R\left(Z_{\mathrm{H}-}\right.$ uncor $)$ estimates, the two types of $R\left(K_{\mathrm{DP}}\right)$ estimates do not show the severe underestimation and show better agreement with the gauge measurements. This result supports the fact that $K_{\mathrm{DP}}$ is not affected by attenuation (Ryzhkov and Zrnic 1995). However, the $R\left(K_{\mathrm{DP}}\right)$ estimates combined with

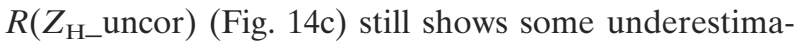
tion at large rainfall accumulations above about $10 \mathrm{~mm}$, with NB of $-12.2 \%$. On the contrary, the $R\left(K_{\mathrm{DP}}\right)$ estimates combined with $R\left(Z_{\mathrm{H}-}\right.$ cor $)$ show good agreement with the gauge measurements, with an NE and NB of $14.8 \%$ and $-1.1 \%$, respectively. Further, this estimation error is smaller than that of the $R\left(Z_{\mathrm{H}-}\right.$ uncor) estimates. This decrease of estimation error can be attributed to the fact that $R\left(K_{\mathrm{DP}}\right)$ estimates are less sensitive to natural variations of DSDs than $R\left(Z_{\mathrm{H}}\right)$ estimates (Ryzhkov and Zrnic 1996).

Similar variation patterns of the estimation errors to those for the 1-h rainfall accumulations are also shown for other time intervals, that is, $15 \mathrm{~min}$ and $3 \mathrm{~h}$ (Table 2). Namely, the correction of $Z_{\mathrm{H}}$ for attenuation produces reliable rainfall amounts, but more accurate rainfall amounts is shown in the $R\left(K_{\mathrm{DP}}\right)$ estimates combined with $R\left(Z_{\mathrm{H}-c o r}\right)$, because $K_{\mathrm{DP}}$ is less sensitive to DSD variations. As expected, the estimation errors for the various rainfall estimates decreases with an increasing time interval. In summary, it is concluded that $K_{\mathrm{DP}}$ can produce more accurate rainfall estimation because of its advantages, such as independency on attenuation and less sensitivity to DSD variations, but the rainfall estimates using $K_{\mathrm{DP}}$ should be superseded by the estimates from the corrected $Z_{\mathrm{H}}$ for attenuation, for weak rainfall rates, in order to improve the accuracy of the radar-rainfall estimation. In addition, rainfall amounts with good accuracy can be derived from radar data at $\mathrm{X}$ band if correction for attenuation is done accurately.

In the experiments of rainfall estimation above, the $R\left(Z_{\mathrm{H}}\right)$ estimator derived from the disdrometer data in 2001 was applied to the radar measurements in 2003.
Because the $R\left(Z_{\mathrm{H}}\right)$ estimator is sensitive to spatial and temporal variations in DSD, it may be more representative to derive a new $R\left(Z_{\mathrm{H}}\right)$ estimator in order to apply to the radar data in 2003. As mentioned earlier, however, it is generally difficult to adjust the $R\left(Z_{\mathrm{H}}\right)$ estimator to a new environment accompanied with spatial and temporal changes of DSD, in particular, in the operational monitoring of rainfall, which has been one purpose of our experiment since 2003. Indeed, $R\left(Z_{\mathrm{H}}\right)$ estimators continuously change with time and space, even within a rain cell (Bringi et al. 2004). Therefore, the $R\left(Z_{\mathrm{H}}\right)$ estimator in 2001 was assumed as a general form and applied to the radar measurements in 2003. As shown above, the $R\left(Z_{\mathrm{H}}\right)$ estimator produced good agreement with gauge measurements. The scattering simulations based on the disdrometer data in 2001 and 2003 show $R\left(Z_{\mathrm{H}}\right)=6.84 \times 10^{-2} Z_{\mathrm{H}}^{0.585}$ for $Z_{\mathrm{H}}>35$ $\mathrm{dB} Z$, which produces somewhat larger rainfall rates than the estimator in 2001 (2a). In contrast to the $R\left(Z_{\mathrm{H}}\right)$ estimator, the $R\left(K_{\mathrm{DP}}\right)$ estimator does not show such variability. The scattering simulations based on the disdrometer data in 2001 and 2003 show $R\left(K_{\mathrm{DP}}\right)=$ $19.93 K_{\mathrm{DP}}^{0.827}$, which produces almost the same rainfall rates as by the estimator in (2b). Thus, the $R\left(K_{\mathrm{DP}}\right)$ estimator is less sensitive to DSD variations.

The rainfall estimates shown above were derived from the relation (2) based on the Andsager relation for drop shapes in the scattering simulations. Because $K_{\mathrm{DP}}$ is largely affected by variation of the drop shapes, the multiplicative coefficient of the $R\left(K_{\mathrm{DP}}\right)$ estimator also changes with the assumed relations for drop shapes, and, consequently, rainfall estimation based on $K_{\text {DP }}$ is also affected (Gorgucci et al. 2000; Matrosov et al. 2002). When the Keenan and minimum relations are assumed in the scattering simulations, $R\left(K_{\mathrm{DP}}\right)$ estimators are $R\left(K_{\mathrm{DP}}\right)=20.90 K_{\mathrm{DP}}^{0.841}$ and $R\left(K_{\mathrm{DP}}\right)=13.21 K_{\mathrm{DP}}^{0.894}$, respectively. Meanwhile, the $R\left(Z_{\mathrm{H}}\right)$ estimators are, as expected, nearly constant with respect to drop shapes. When the Keenan and minimum relations are employed for rainfall estimation using the same data as in Fig. 14, the $R\left(K_{\mathrm{DP}}\right)$ estimates, combined with the $R\left(Z_{\mathrm{H}-}\right.$ cor $)$ estimates for the Keenan relation, produce an NE and NB of $15.4 \%$ and $3.5 \%$, respectively, while those for the minimum relation produce an NE and NB of $27.1 \%$ and $-24.8 \%$, respectively. Thus, the $R\left(K_{\mathrm{DP}}\right)$ estimator for the Andsager relation produced the most accurate rainfall amounts for the data used in this study. On the other hand, Matrosov et al. (2002) showed, using the procedure of Gorgucci et al. (2000) modified for $\mathrm{X}$ band, that the equilibrium drop shape relation of Pruppacher and Beard (1970) was suitable for most data obtained in their experiment. Therefore, it is suggested that use of the $R\left(K_{\mathrm{DP}}\right)$ estimator requires careful 
attention in the selection of the drop shape model. It is possible to estimate an effective slope of a linear drop shape model using the methodology of Gorgucci et al. (2000) at X band to "correct" the multiplicative coefficient of the $R\left(K_{\mathrm{DP}}\right)$ estimator. This procedure, which uses $Z_{\mathrm{H}}, Z_{\mathrm{DR}}$, and $K_{\mathrm{DP}}$, is used in the following section to estimate the DSD parameters (when $K_{\mathrm{DP}}$ and $Z_{\mathrm{DR}}$ are not "noisy"), but is not used in this section for rainfall comparison with gauges.

\section{b. Estimation of raindrop size distribution parameters}

Estimation of the raindrop size distribution is important for studying the evolution of rainfall microphysical processes, especially the vertical structure of the DSD. It is difficult to attempt at X band wavelength because the $Z_{\mathrm{DR}}$ (which is an important measure of the reflectivity-weighted mean drop diameter) has to be accurately corrected for differential attenuation. Similarly, the $Z_{\mathrm{H}}$ is used for estimating the drop concentration, and its correction for rain attenuation also has to be done accurately. A further complication is that the drop shape model has to be either assumed or derived from the corrected data itself. Here, the two parameters of a normalized gamma DSD are estimated from the corrected $Z_{\mathrm{H}}$, corrected $Z_{\mathrm{DR}}$, and $K_{\mathrm{DP}}$ using the algorithm developed by Gorgucci et al. (2002a,b) adapted for X band. The two parameters of the DSD are the median volume diameter $\left(D_{0}\right)$ and the normalized intercept parameter $N_{w}$ (or $N_{0}^{*}$ or $N_{L}$ ) as defined in Testud et al. (2000) and Illingworth and Blackman (2002). The intent here is to evaluate the retrieval of these parameters in one event (a typhoon on 11 September 2001) with moderate-to-heavy rainfall rates $\left(R>10 \mathrm{~mm} \mathrm{~h}^{-1}\right)$ and compare the distributions with representative distributions from DSDs measured with disdrometers. It provides a basis for (indirectly) evaluating the overall calibration of the radar as well as the accuracy of the attenuation-correction methodology (Le Bouar et al. 2001).

According to the algorithm of Gorgucci et al. (2002a) for X-band measurements, the two DSD parameters $D_{0}$ $(\mathrm{mm})$ and $N_{w}\left(\mathrm{~mm}^{-1} \mathrm{~m}^{-3}\right)$ are estimated by the following relations:

$$
\begin{aligned}
D_{0} & =0.627 Z_{\mathrm{H}}^{0.057} \xi_{\mathrm{DR}}^{\left(0.03 \beta_{\mathrm{eff}}^{-1.22}\right)}, \\
\log _{10} N_{w} & =2.97 Z_{\mathrm{H}}^{0.070} \xi_{\mathrm{DR}}^{\left(-0.03 \beta_{\mathrm{eff}}^{-1.26}\right)}
\end{aligned}
$$

[where $Z_{\mathrm{H}}$ is in $\mathrm{mm}^{6} \mathrm{~m}^{-3}$ and $\xi_{\mathrm{DR}}$ is the linear scale of $\left.Z_{\mathrm{DR}}\left(Z_{\mathrm{DR}}=10 \log _{10} \xi_{\mathrm{DR}}\right)\right]$. In the above relations, $\beta_{\mathrm{eff}}$ is in $\mathrm{mm}^{-1}$ and denotes an "effective slope" of the relation between drop axis ratio $(a / b)$ and drop diameter
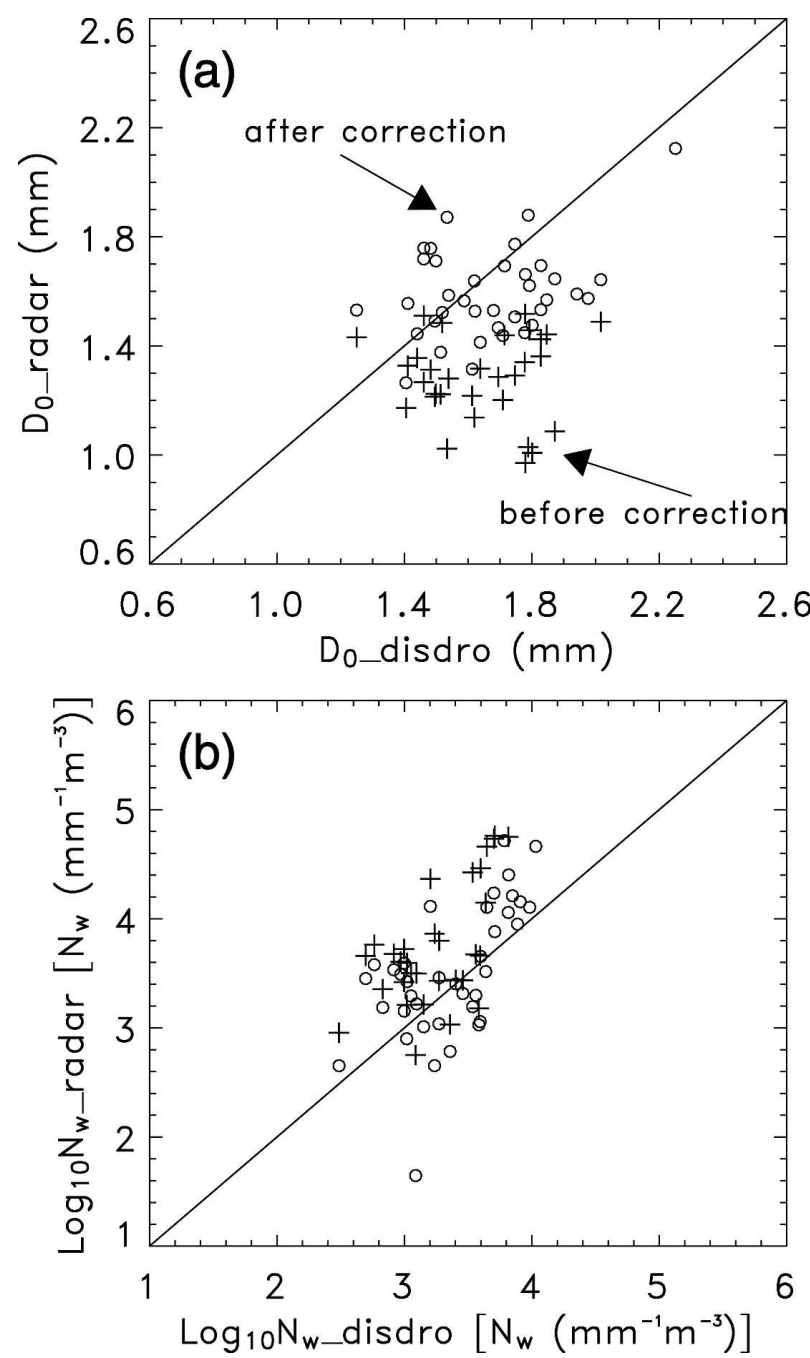

FIG. 15. Scatterplots of the normalized DSD parameters (a) $D_{0}$ and (b) $\log _{10} N_{w}$ between disdrometer and radar estimates, from 0150 to 0220 LST 11 Sep 2001.

(D) (i.e., $a / b=1-\beta_{\mathrm{eff}} D$ ). One key concept of the estimation algorithm is that the drop shape model is not fixed by a preassumed relation, but is estimated by $\beta_{\text {eff }}$. Gorgucci et al. (2000) showed that $\beta_{\text {eff }}$ can be determined from the polarimetric dataset $\left(Z_{\mathrm{H}}, Z_{\mathrm{DR}}\right.$, and $\left.K_{\mathrm{DP}}\right)$. In this study, $\beta_{\text {eff }}$ was estimated as follows:

$$
\beta_{\text {eff }}=0.9425\left(K_{\mathrm{DP}} / Z_{\mathrm{H}}\right)^{0.2624}\left(\xi_{\mathrm{DR}}-1\right)^{0.377} .
$$

The above form for $\beta_{\text {eff }}$ was shown by Bringi et al. (2004) to be derivable from the first principles assuming Rayleigh scattering. The coefficients and exponents in (6) for $\mathrm{X}$ band are obtained from scattering simulations.

Figure 15 shows comparisons of $D_{0}$ and $N_{w}$ between disdrometer and radar estimates over the three disdrometer sites from 0150 to 0220 LST 11 September 
2001, when the strong echo with high $Z_{\mathrm{H}}$ and $Z_{\mathrm{DR}}$ occurred as shown in Fig. 7. Note that the DSD estimation algorithm described above is the one applicable for moderate-to-heavy rainfall rates, because otherwise $Z_{\mathrm{DR}}$ and $K_{\mathrm{DP}}$ are "noisy," precluding an accurate estimation of $\beta_{\mathrm{eff}}$. For retrieval of the DSD parameters from radar data, first $Z_{\mathrm{H}}$ and $Z_{\mathrm{DR}}$ were smoothed over the same range interval as used in the $K_{\mathrm{DP}}$ estimation. The DSD parameters from the disdrometer measurements were averaged for $3 \mathrm{~min}$, for comparison with radar estimates with an interval of $3 \mathrm{~min}$. As shown in the figure, the $D_{0}$ estimates (plus signs) from the uncorrected $Z_{\mathrm{H}}$ and $Z_{\mathrm{DR}}$, and $K_{\mathrm{DP}}$ present significant underestimation (Fig. 15a), with the corresponding NE and NB of $22.6 \%$ and $-21.7 \%$, respectively (Table 3 ). After attenuation correction (circles), a good agreement with those from the disdrometer measurements is shown, in which the corresponding NE and NB were largely reduced to $11.2 \%$ and $-4.8 \%$, respectively. The comparison of $\log _{10} N_{w}$ estimates (Fig. 15b) also shows a similar pattern: the $\log _{10} N_{w}$ estimates from the uncorrected radar data present overestimation, resulting from low $Z_{\mathrm{H}}$ and $Z_{\mathrm{DR}}$ caused by attenuation, while the corrected radar data produce the $\log _{10} N_{w}$ estimates in good agreement with those from the disdrometer measurements. The accuracy of the DSD estimation shown in the present study may be improved through a higher performance algorithm; however, the intent of this study is to demonstrate that X-band polarimetric radar can be used to extract DSD information if the attenuation is well corrected. An improvement of the DSD estimation scheme, therefore, is a subject of future work.

Figure 16 shows a comparison of the frequency of occurrence or relative frequency histogram of $D_{0}$ and $N_{w}$ between the radar estimates and disdrometer measurements. The DSD estimation algorithm was applied to only data with rainfall rates above $10 \mathrm{~mm} \mathrm{~h}^{-1}$ and $Z_{\mathrm{H}}$ above $35 \mathrm{~dB} Z$, because otherwise $Z_{\mathrm{DR}}$ and $K_{\mathrm{DP}}$ are noisy, precluding an accurate estimation of $\beta_{\text {eff }}$. In Fig. 16 , the radar retrievals are from the typhoon event from 0100 to 0300 LST 11 September 2001, while the disdrometer dataset is based on all rain events from June to December 2001 in the Tsukuba area of Japan. Even though the radar data are from a single event over a 2-h period, the large spatial sampling of rain cells in different stages with their evolution tends to capture a wide range of DSD variability (see, also, Bringi et al. 2003). As shown in Fig. 16a, the $D_{0}$ histogram obtained from the uncorrected $Z_{\mathrm{H}}$ and $Z_{\mathrm{DR}}$ gives smaller $D_{0}$ values than those from the disdrometer measurements. After correction, the $D_{0}$ histogram shows quite good agreement with that from the disdrometer measurements.
TABLE 3. The NE and NB of the DSD parameters $D_{0}$ and $\log _{10} N_{w}$ estimated from radar data.

\begin{tabular}{lccccc}
\hline \hline & \multicolumn{2}{c}{$D_{0}$} & & \multicolumn{2}{c}{$\log _{10} N_{w}$} \\
\cline { 2 - 3 } \cline { 5 - 6 } & $\begin{array}{c}\text { Before } \\
\text { correction }\end{array}$ & $\begin{array}{c}\text { After } \\
\text { correction }\end{array}$ & & $\begin{array}{c}\text { Before } \\
\text { correction }\end{array}$ & $\begin{array}{c}\text { After } \\
\text { correction }\end{array}$ \\
\hline NE (\%) & 22.6 & 11.2 & & 17.2 & 11.9 \\
NB (\%) & -21.7 & -4.8 & & 15.0 & 4.1 \\
\hline
\end{tabular}

The modal value occurs at $1.4 \mathrm{~mm}$ for both datasets. In the case of the $N_{w}$ histogram, the distribution from the uncorrected data gives an overestimation. The uncorrected radar estimates give a modal value at $\log _{10}\left(N_{w}\right)$ $=4.6$, while the disdrometer measurements gives the mode at 4.0 (note that the Marshall-Palmer $N_{w}$ for exponential DSD is $8000 \mathrm{~mm}^{-1} \mathrm{~m}^{-3}$ or 3.9 in log scale). After correction, the $N_{w}$ distribution is very similar to that from the disdrometer measurements with similar modal values. The smaller $D_{0}$ and larger $N_{w}$ values obtained from the uncorrected $Z_{\mathrm{H}}$ and $Z_{\mathrm{DR}}$ values are obviously the result of attenuation effects. The good agreement of $D_{0}$ and $N_{w}$ histograms retrieved from the corrected radar data as compared with disdrometer data shows that the radar was well calibrated and that the correction algorithm for $Z_{\mathrm{H}}$ and $Z_{\mathrm{DR}}$ attenuation worked fairly well. In fact, Le Bouar et al. (2001) suggest "fine tuning" the reflectivity calibration offset to ensure that the mode of $\log _{10}\left(N_{w}\right)$ from radar matches the corresponding mode from the disdrometer data.

\section{Summary and conclusions}

In this paper, the self-consistent method for correction of rain attenuation is modified to the $\mathrm{X}$-band polarimetric measurements, based on the results obtained from scattering simulations described in Part I. The modified attenuation-correction methodology is evaluated with radar measurements from the MP-X radar of NIED. To validate the correction method, the corrected radar data are compared with scattering simulations using ground-based disdrometer data. Further, effects of attenuation on estimation of rainfall amounts and drop size distribution parameters are investigated, by comparing the radar estimates after and before correction with ground-based gauge and disdrometer measurements.

The corrected $Z_{\mathrm{H}}$ and $Z_{\mathrm{DR}}$ for attenuation were in good agreement with values simulated from groundbased disdrometer data, over three different sites. Attenuation $A_{\mathrm{H}}$ and $A_{\mathrm{DP}}$ derived in the correction procedure also agreed well with the simulated values. In addition, the corrected data were shown to be consistent with empirical relations among the polarimetric vari- 
$R>10 \mathrm{~mm} \mathrm{~h}^{-1} \& \mathrm{Z}_{\mathrm{H}}>35 \mathrm{dBZ}$
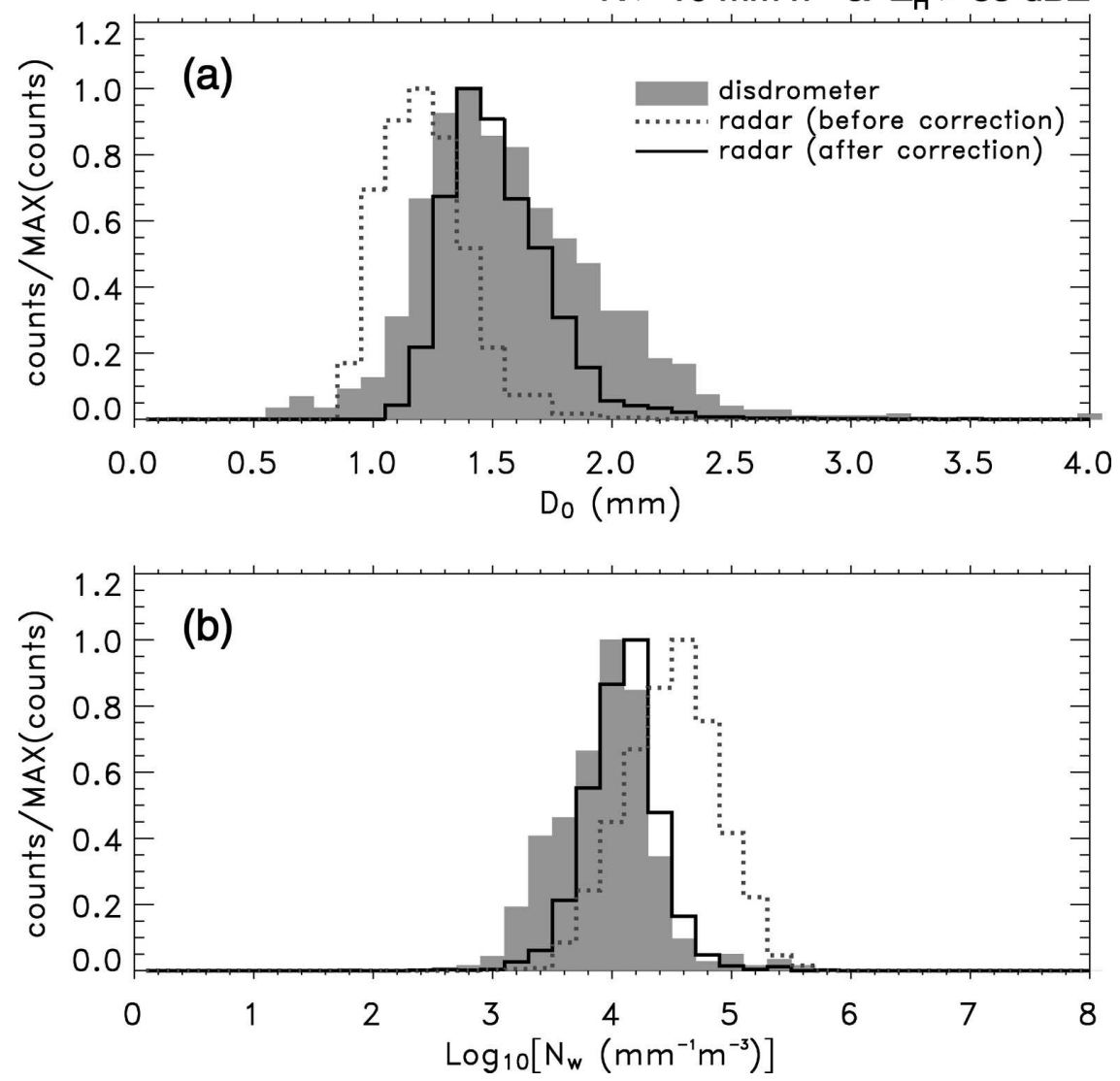

FIG. 16. Histograms of (a) $D_{0}$ and (b) $\log _{10} N_{w}$ for rainfall rates above $10 \mathrm{~mm} \mathrm{~h}^{-1}$ and radar reflectivity factors above $35 \mathrm{dBZ}$. The histogram from disdrometer is based on drop spectra measured from Jun to Dec 2001. The histogram from radar is based on data from 0100 to 0300 LST 11 Sep 2001.

ables derived from scattering simulations (e.g., $K_{\mathrm{DP}^{-}}$ $Z_{\mathrm{H}}, A_{\mathrm{H}}-Z_{\mathrm{H}}, Z_{\mathrm{DR}}-Z_{\mathrm{H}}, A_{\mathrm{DP}}-A_{\mathrm{H}}$ relations). The accuracy of the attenuation correction highly depends on the determination of optimal values of the coefficients $\alpha$ and $\gamma$ of the $A_{\mathrm{H}}-K_{\mathrm{DP}}$ and $A_{\mathrm{DP}}-A_{\mathrm{H}}$ relations, respectively. It was shown in this study that the optimal $\alpha$ and $\gamma$ values determined from radar data were in good consistency with their empirical relationship obtained from scattering simulations. This good agreement suggests that the data measured by the MP-X radar were well corrected for attenuation by the modified self-consistent method.

Further, we examined effects of attenuation on the estimation of rainfall amounts and raindrop size distributions. In comparisons of 1 -h rainfall accumulations with gauge measurements, the radar estimates $R\left(Z_{\mathrm{H}-}\right.$ uncor) from the uncorrected (observed) $Z_{\mathrm{H}}$ produced severe underestimation with a normalized error (NE) and normalized bias (NB) of $48.9 \%$ and $-47.6 \%$, respectively, while the $R\left(Z_{\mathrm{H}_{-}}\right.$cor $)$estimates from the corrected $Z_{\mathrm{H}}$ showed better agreement with the gauge measurements, with an NE and NB of $19.1 \%$ and $-2.0 \%$, respectively. More accurate rainfall amounts were obtained from a simple $R\left(K_{\mathrm{DP}}\right)$ estimator applied when $K_{\mathrm{DP}}>0.3^{\circ} \mathrm{km}^{-1}$ and $Z_{\mathrm{H}}>35 \mathrm{dBZ}$; otherwise, the $R\left(Z_{\mathrm{H}-}\right.$ cor $)$ estimates were used. This composite algorithm resulted in the smallest NE and NB of $14.8 \%$ and $-1.1 \%$, respectively. This improvement of rainfall estimation by $R\left(K_{\mathrm{DP}}\right)$ is the result of less sensitivity to natural variations of DSDs. In the case of the retrieval of DSDs, the two parameters of the normalized gamma DSD function (i.e., the median volume diameter $D_{0}$ and the intercept parameter $N_{w}$ ) retrieved from the uncorrected radar data presented large deviations from the disdrometer data. The normalized biases of $D_{0}$ and $\log _{10} N_{w}$ were $-21.7 \%$ and $15.0 \%$, respectively. For the corrected radar data, meanwhile, these large deviations were reduced to $-4.8 \%$ and $4.1 \%$ for $D_{0}$ and $\log _{10} N_{w}$, respectively. The normalized error of $D_{0}\left(\log _{10} N_{w}\right)$ was also largely reduced from $22.6 \%(17.2 \%)$ to $11.2 \%$ 
(11.9\%), owing to attenuation correction. In addition, the histograms of $D_{0}$ and $\log _{10} N_{w}$ retrieved from the corrected $Z_{\mathrm{H}}$ and $Z_{\mathrm{DR}}$, and $K_{\mathrm{DP}}$ data showed quite good agreement with corresponding histograms from disdrometer data.

As summarized above, a polarimetric radar at $\mathrm{X}$ band can provide valuable information for hydrometeorological studies, such as rainfall amounts and DSDs, if attenuation is well corrected. In the present study, we considered the simple rainfall estimation algorithm using $R\left(Z_{\mathrm{H}}\right)$ and $R\left(K_{\mathrm{DP}}\right)$. In addition, these rainfall estimators were derived for a preassumed relation for drop shapes [i.e., the relations recommended by Andsager et al. (1999) for $1 \leq D \leq 4.4 \mathrm{~mm}$ and the equilibrium shapes of Beard and Chuang (1987) for $D$ $<1$ or $D>4.4 \mathrm{~mm}$ ]. Although this composite relation was found to be the most suitable in this study as compared with other models, the approach of Gorgucci et al. (2001), as applied by Matrosov et al. (2002), needs further investigation at $\mathrm{X}$ band. Their rainfall algorithm is constructed to be relatively immune to the selection of the drop shape model, but does demand that attenuation correction be very precise because $Z_{\mathrm{H}}$ and $Z_{\mathrm{DR}}$ are used along with $K_{\mathrm{DP}}$. Similarly, algorithms for estimating the parameters of a normalized gamma DSD have been developed by Gorgucci et al. (2002a), which have so far not been applied to the X-band radar data. In the present study, $D_{0}$ and $N_{w}$ estimates were performed for the 11 September 2001 typhoon event for $R$ $>10 \mathrm{~mm} \mathrm{~h}^{-1}$, where the $K_{\mathrm{DP}}$ and $Z_{\mathrm{DR}}$ data are reliable. Histograms of radar-derived $D_{0}$ and $N_{w}$ agreed well with histograms from disdrometer data. For weaker rainfall rates with smaller and "noisier" $K_{\mathrm{DP}}$ and $Z_{\mathrm{DR}}$ values, another retrieval algorithm needs to be developed for the X-band radar data, similar to that at $\mathrm{S}$ band proposed by Bringi et al. (2002).

The MP-X radar has been operated at Ebina since 2003 for testing its operational use for monitoring and forecasting heavy rainfall that frequently results in floods and landslides. The radar site is good for these applications because two different topographies consisting of the mountainous area around Mt. Fuji and the flat metropolitan area centered around Tokyo are included in the observable range of the MP-X radar (Maki et al. 2005). The observations will be used for hydrometeorological studies using the MP-X radar, such as the improvement of rainfall estimation, retrieval of DSDs, and classification of hydrometeor types with high spatial and temporal resolution.

Acknowledgments. The authors would like to acknowledge R. Misumi, K. Maruyama, and M. Suto of NIED for supporting radar observations. One of the total three disdrometers was provided by Prof. H. Uyeda of Nagoya University, Japan. The eight rain gauges were provided by Prof. D.-I. Lee of Pukyong University, Korea. The Daito Dengyo Co., is also appreciated for providing space for the radar equipment during the MP-X observations since 2003. This research is supported by the NSF Engineering Research center Program ERC-0313747 (VNB and VC). One of the authors (VNB) acknowledges support from the National Science Foundation via Grant ATM-0140350.

\section{REFERENCES}

Andsager, K., K. V. Beard, and N. F. Laird, 1999: Laboratory measurements of axis ratios for large raindrops. J. Atmos. Sci., 56, 2673-2683.

Beard, K. V., and C. Chuang, 1987: A new model for the equilibrium shape of raindrops. J. Atmos. Sci., 44, 1509-1524.

Bringi, V. N., and V. Chandrasekar, 2001: Polarimetric Doppler Weather Radar: Principles and Applications. Cambridge University Press, $636 \mathrm{pp}$.

- T. D. Keenan, and V. Chandrasekar, 2001: Correcting Cband radar reflectivity and differential reflectivity data for rain attenuation: A self-consistent method with constraints. IEEE Trans. Geosci. Remote Sens., 39, 1906-1915.

_, G.-J. Huang, V. Chandrasekar, and E. Gorgucci, 2002: A methodology for estimating the parameters of a gamma raindrop size distribution model from polarimetric radar data: Application to a squall-line event from the TRMM/Brazil campaign. J. Atmos. Oceanic Technol., 19, 633-645.

— , V. Chandrasekar, J. Hubbert, E. Gorgucci, W. L. Randeu, and M. Schoenhuber, 2003: Raindrop size distribution in different climatic regimes from disdrometer and dual-polarized radar analysis. J. Atmos. Sci., 60, 354-365.

— , T. Tang, and V. Chandrasekar, 2004: Evaluation of a new polarimetrically based $Z-R$ relation. J. Atmos. Oceanic Technol., 21, 612-623.

Doviak, R., V. N. Bringi, A. Ryzhkov, A. Zahari, and D. Zrnic, 2000: Considerations for polarimetric upgrades to operational WSR-88D radar. J. Atmos. Oceanic Technol., 17, 257-278.

Gorgucci, E., G. Scarchilli, and V. Chandrasekar, 1999: A procedure to calibrate multiparameter weather radar using properties of the rain medium. IEEE Trans. Geosci. Remote Sens., 37, 269-276.

,,--- , and V. N. Bringi, 2000: Measurement of mean raindrop shape from polarimetric radar observations. J. Atmos. Sci., 57, 3406-3413.

,,,--- and,- 2001 : Rainfall estimation from polarimetric radar measurements: Composite algorithms immune to variability in raindrop shape-size relation. J. Atmos. Oceanic Technol., 18, 1773-1786.

— , V. Chandrasekar, and V. N. Bringi, 2002a: Drop size distribution retrieval from polarimetric radar measurements. Proc. Second European Conf. on Radar Meteorology, Delft, Netherlands, European Meteorological Society, 134-139.

$-, \ldots,-\ldots$, and G. Scarchilli, 2002b: Estimation of raindrop size distribution parameters from polarimetric radar variables. J. Atmos. Sci., 59, 2373-2384.

Holt, A. R., V. N. Bringi, and D. Brunkow, 1999: A comparison between parameters obtained with the CSU-CHILL radar from simultaneous and switched transmission of vertical and horizontal polarization. Preprints, 29th Int. Conf. on Radar 
Meteorology, Montreal, QC, Canada, Amer. Meteor. Soc., 214-217.

Hubbert, J., and V. N. Bringi, 1995: An iterative filtering technique for the analysis of copolar differential phase and dualfrequency polarimetric variables. J. Atmos. Oceanic Technol., 12, 643-648.

_ , V. Chandrasekar, V. N. Bringi, and P. Meischner, 1993: Processing and interpretation of coherent dual-polarized polarimetric variables. J. Atmos. Oceanic Technol., 10, 155-164.

Illingworth, A. J., and T. M. Blackman, 2002: The need to present raindrop size spectra as normalized gamma distributions for the interpretation of polarization radar observations. J. Appl. Meteor., 41, 286-297.

Iwanami, K., R. Misumi, M. Maki, T. Wakayama, K. Hata, and S. Watanabe, 2001: Development of a multiparameter radar system on mobile platform. Preprints, 30th Int. Conf. on Radar Meteorology, Munich, Germany, Amer. Meteor. Soc., 104-106.

, E. Le Bouar, J. Testud, M. Maki, R. Misumi, S.-G. Park, and M. Suto, 2003: Application of the rain profiling algorithm $\mathrm{ZPHI}$ to the X-band polarimetric radar data observed in Japan. Preprints, 31st Int. Conf. on Radar Meteorology, Seattle, WA, Amer. Meteor. Soc., 274-276.

Le Bouar, E., J. Testud, and T. D. Keenan, 2001: Validation of the rain profiling algorithm $\mathrm{ZPHI}$ from the C-band polarimetric weather radar in Darwin. J. Atmos. Oceanic Technol., 18, 1819-1837.

Liu, L., V. N. Bringi, I. J. Caylor, and V. Chandrasekar, 1993: Intercomparison of multiparameter radar signatures from Florida storms. Preprints, 26th Int. Conf. on Radar Meteorology, Norman, OK, Amer. Meteor. Soc., 733-735.

Maki, M., and Coauthors, 2005: Semi-operational rainfall obser- vations with X-band multi-parameter radar. Atmos. Sci. Lett., 6, 12-18.

Matrosov, S. Y., K. A. Clark, B. E. Martner, and A. Tokay, 2002: $\mathrm{X}$-band polarimetric radar measurements of rainfall. J. Appl. Meteor., 41, 941-952.

Park, S.-G., V. N. Bringi, V. Chandrasekar, M. Maki, and K. Iwanami, 2005: Correction of radar reflectivity and differential reflectivity for rain attenuation at X-band. Part I: Theoretical and empirical basis. J. Atmos. Oceanic Technol., 22, 16211632.

Pruppacher, H. R., and K. V. Beard, 1970: A wind tunnel investigation of the internal circulation and shape of water drops falling at terminal velocity in air. Quart. J. Roy. Meteor. Soc., 96, 247-256.

Ryzhkov, A., and D. S. Zrnic, 1995: Precipitation and attenuation measurements at a $10-\mathrm{cm}$ wavelength. J. Appl. Meteor., 34, 2121-2134.

— , and _ 1996: Assessment of rainfall measurement that uses specific differential phase. J. Appl. Meteor., 35, 20802090.

— and _- 1998: Polarimetric rainfall estimation in the presence of anomalous propagation. J. Atmos. Oceanic Technol., 15, 1320-1330.

Testud, J., E. Le Bouar, E. Obligis, and M. Ali-Mehenni, 2000: The rain profiling algorithm applied to polarimetric weather radar. J. Atmos. Oceanic Technol., 17, 332-356.

Zawadzki, I. I., 1975: On radar-raingage comparison. J. Appl. Meteor., 14, 1430-1436.

Zrnic, D. S., and A. V. Ryzhkov, 1996: Advantages of rain measurements using specific differential phase. J. Atmos. Oceanic Technol., 13, 464-476. 